\title{
De novo identification of universal cell mechanics regulators
}

Marta Urbanska1,2,, Yan Ge ${ }^{1, t, \S}$, Maria Winzi ${ }^{1}$, Shada Abuhattum ${ }^{1,2}$, Maik Herbig ${ }^{1,2,3}$, Martin Kräter ${ }^{1,2}$, Nicole Toepfner ${ }^{1,4}$, Joanne Durgan ${ }^{5}$, Oliver Florey ${ }^{5}$, Martina Dori ${ }^{3}$, Federico Calegari ${ }^{3}$, Fidel-Nicolás Lolo ${ }^{6}$, Miguel Ángel del Pozo $^{6}$, Anna Taubenberger ${ }^{1}$, Carlo Vittorio Cannistraci ${ }^{1,7,8,9 *}$, Jochen Guck ${ }^{1,2, *}$

${ }^{1}$ Biotechnology Center, Center for Molecular and Cellular Bioengineering, Technische Universität Dresden, Tatzberg 47-49, Dresden 01307, Germany

${ }^{2}$ Max Planck Institute for the Science of Light \& Max-Planck-Zentrum für Physik und Medizin, Staudtstraße 2, Erlangen 91058, Germany

${ }^{3}$ Center for Regenerative Therapies Dresden, Center for Molecular and Cellular Bioengineering, Technische Universität Dresden, Fetscherstrasse 105, Dresden 01307, Germany

${ }^{4}$ Klinik und Poliklinik für Kinder- und Jugendmedizin, Universitätsklinikum Carl Gustav Carus, Technische Universität Dresden, Fetscherstraße 74, Dresden 01307, Germany

${ }^{5}$ Signalling Programme, The Babraham Institute, Babraham Research Campus, Cambridge CB22 3AT, UK

${ }^{6}$ Mechanoadaptation and Caveolae Biology lab, Cell and Developmental Biology Area, Centro Nacional de Investigaciones Cardiovasculares (CNIC), Calle de Melchor Fernández Almagro 3, Madrid 28029, Spain

${ }^{7}$ Center for Complex Network Intelligence at the Tsinghua Laboratory of Brain and Intelligence, Department of Bioengineering, Tsinghua University, Chengfu Rd. 160, SanCaiTang Building, Haidian District, Beijing 100084, China

${ }^{8}$ Center for Systems Biology Dresden, Pfotenhauerstraße 108, Dresden 01307, Germany

${ }^{9}$ Cluster of Excellence Physics of Life, Technische Universität Dresden, Tatzberg 47-49, Dresden 01307, Germany

${ }^{\dagger}$ Current address: Institute for Immunology, Universitätsklinikum Carl Gustav Carus, Technische Universität Dresden, Dresden, Germany

${ }^{\S}$ These authors contributed equally to this work

${ }^{*}$ Corresponding authors: kalokagathos.agon@gmail.com, jochen.guck@mpl.mpg.de 


\section{Abstract}

Mechanical proprieties determine many cellular functions, such as cell fate specification, migration, or circulation through vasculature. Identifying factors governing cell mechanical phenotype is therefore a subject of great interest. Here we present a mechanomics approach for establishing links between mechanical phenotype changes and the genes involved in driving them. We employ a machine learning-based discriminative network analysis method termed PC-corr to associate cell mechanical states, measured by real-time deformability cytometry (RT-DC), with large-scale transcriptome datasets ranging from stem cell development to cancer progression, and originating from different murine and human tissues. By intersecting the discriminative networks inferred from two selected datasets, we identify a conserved module of five genes with putative roles in the regulation of cell mechanics. We validate the power of the individual genes to discriminate between soft and stiff cell states in silico, and demonstrate experimentally that the top scoring gene, CAV1, changes the mechanical phenotype of cells when silenced or overexpressed. The data-driven approach presented here has the power of de novo identification of genes involved in cell mechanics regulation and paves the way towards engineering cell mechanical properties on demand to explore their impact on physiological and pathological cell functions.

Keywords: mechanical phenotype, deformability cytometry, transcriptomics, RNAseq, unsupervised machine learning, systems biology, discriminative network analysis, mechanomics 


\section{Introduction}

The extent to which cells can be deformed by external loads is determined by their mechanical properties, such as cell stiffness. Since the mechanical phenotype of cells has been shown to reflect functional cell changes, it is now well established as a sensitive labelfree biophysical marker of cell state in health and disease $\mathrm{e}^{1-4}$. Beyond being a passive property that can be correlated with cell state, cell stiffness is increasingly recognized as an important feature involved in shaping processes such as development ${ }^{5-8}$ and cancer progression ${ }^{9-13}$. Identifying the molecular targets for on-demand tuning of mechanical properties is, thus, essential for exploring the precise impact that cell mechanics has on physiological and pathological processes in living organisms.

The mechanical properties of cells are determined by various intracellular structures and their dynamics, with cytoskeletal networks at the forefront ${ }^{14}$. According to current knowledge, the most prominent contributor to the global mechanical phenotype is the actin cortex and its contractility regulated via Rho signalling ${ }^{15-17}$. Intermediate filaments, including vimentin and keratin, reside deeper inside the cell and can also contribute to measured cell stiffness, especially at high strains ${ }^{18-22}$. Although there is some evidence of the contribution of microtubules to cell stiffness at high strains ${ }^{23}$, their role has been difficult to address directly, since drug-induced microtubule disassembly evokes reinforcement of actin cytoskeleton and cell contractility ${ }^{24}$. Apart from cytoskeletal contributions, the cell mechanical phenotype can be influenced by the level of intracellular packing ${ }^{25-27}$ or mechanical properties of organelles occupying cell interior, such as the cell nucleus ${ }^{28,29}$. When aiming at modulating the mechanical properties of cells, it may not be practical to target cytoskeletal structures, which are central to a multitude of cellular processes, because their disruption is generally toxic to cells. It is therefore important to identify targets that enable subtle, alternative ways of intervening with cell stiffness.

Most of our knowledge about the molecular contributors to cell mechanics has been derived from drug perturbations or genetic modifications targeting structures known a priori. The 
challenge of identifying novel targets determining the mechanical phenotype can be addressed on a large scale by performing screens using RNA interference ${ }^{30-32}$ or smallmolecule compound libraries. Alternatively, the problem can be reverse-engineered, in that omics datasets for systems with known mechanical phenotype changes are used for prediction of genes involved in the regulation of mechanical phenotype in a mechanomics approach $^{4}$. Broadly speaking, mechanomics is a study of omics data within the context of mechanobiology. So far, this term was used with regard to changes in omics profiles in response to a mechanical stimuli such as shear flow, tensile stretch, or mechanical compression ${ }^{33-35}$, or to collectively name all of the mechanical forces acting on or within cells $^{36-40}$. However, it can also be used to address omics changes related to changes in the mechanical properties of cells $s^{41,42}$ - a context much closer to our study.

Here we extend the concept of mechanomics to a data-driven methodology for de novo identification of genes contributing to the mechanical phenotype based on omics data (Figure 1). To demonstrate this approach, we perform a machine learning-based discriminative network analysis termed PC-corr ${ }^{41}$ on transcriptomics data from two unrelated biological systems with known mechanical phenotype changes ${ }^{42,43}$ and elucidate a conserved functional module of five candidate genes putatively involved in the regulation of cell mechanics. We then test the ability of each gene to classify cell states according to cell stiffness in silico on four further datasets. Finally, we verify experimentally that the gene with the highest discriminative power, caveolin 1 (CAV1), has the capacity to alter the mechanical phenotype in the predicted direction when downregulated or overexpressed. The systematic approach presented here, combining omics data with mechanical phenotypes across different systems, has the power to identify genes that ubiquitously contribute to cell mechanical phenotype in a hypothesis-free manner. Such genes can, in the future, be used as knobs for adjusting mechanical cell properties to explore their role in the homeostasis of multicellular systems or to therapeutically intervene in relevant pathologies. 


\section{Results}

\section{Cross-system identification of genes involved in cell mechanical changes}

We introduce an inference approach for de novo identification of genes involved in cell mechanical changes across different systems from transcriptomic data that we refer to as mechanomics. The general workflow of this approach is presented in Figure 1 and consists of four following steps: data curation, target prediction, in silico validation and experimental validation.

a dataset curation

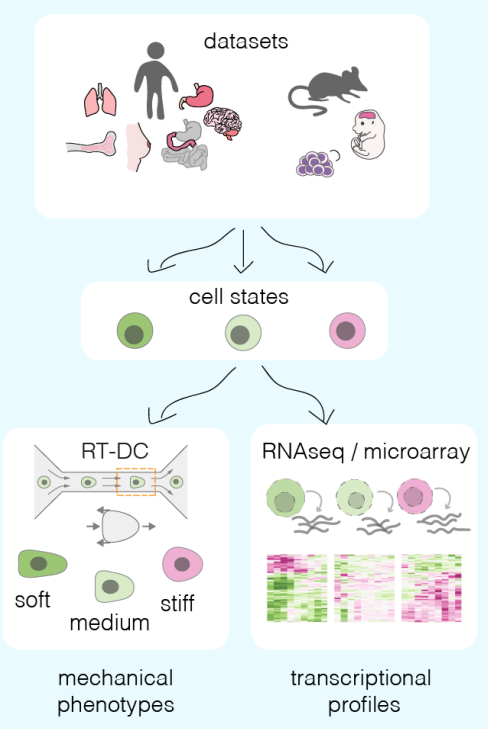

b target prediction
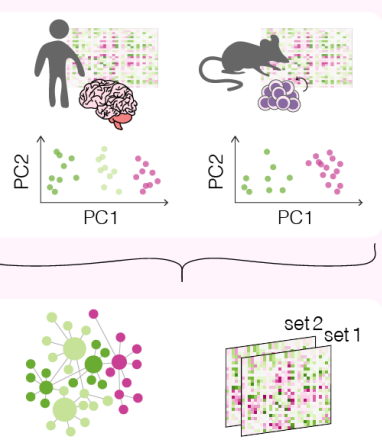

network analysis PC-corr scores

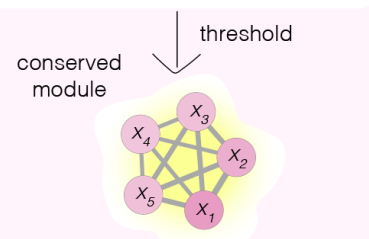

C

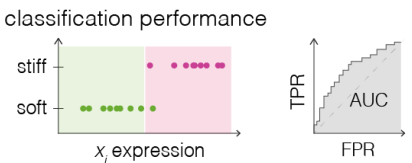

asses across all validation datasets
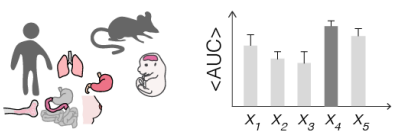

d experimental validation

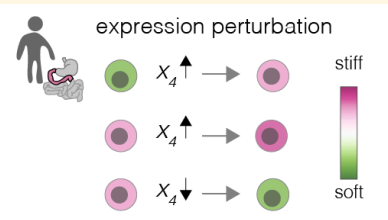

Figure 1 | Overview of a mechanomics approach for the de novo identification of genes involved in cell mechanics regulation. a, Data curation. Datasets originating from different biological systems encompassing cell states with distinct mechanical phenotypes, as characterized by RT-DC, and associated transcriptomics profiles are collected. b, Target prediction. A subset of collected datasets is used to perform machine learning-based network analysis on transcriptomic data and identify conserved module of genes associated with cell mechanics changes. PC - principal component. c, In silico validation. The classification performance of individual genes from module identified in (b) is evaluated in silico on remaining datasets. TPR - true positive rate, FPR - false positive rate, AUC - area under the curve. d, Experimental validation. Targets with highest classification performance in silico are verified experimentally in perturbation experiments. 
In the first step, datasets representing a broad spectrum of biological systems are collected

(Figure 1a). Each dataset encompasses two or more distinct cell states characterized by a change in the mechanical phenotype, and for which transcriptomic data is available. For the particular execution presented in this study, we curated six datasets originating from various mouse and human tissues (Table 1). The mechanical phenotypes of the different cell states within each dataset were characterized using real-time deformability cytometry (RT-DC), a microfluidics-based method that enables rapid analysis of thousands of cells ${ }^{44}-a$ feature particularly useful when setting out to explore a large variety of systems and states. The transcriptional profiles related to each system, generated by either RNA sequencing (RNAseq) or microarray analysis, were retrieved from entries previously deposited in online databases (Table 1).

In the second step, the transcriptomic data from a subset of collected datasets is used to identify a conserved network module of putative target genes involved in the regulation of cell mechanical phenotype (Figure 1b). For this purpose, we implemented an inference approach termed PC-corr ${ }^{41}$, which predicts a network of features that explain the sample segregation along the principal component (PC) associated with a phenotypic change. PC-corr is performed individually on each discovery dataset and the juxtaposition of the results from individual sets yields a conserved module of genes that are the predicted targets potentially involved in the regulation cell mechanics. In our study, we have included two datasets from unrelated systems in the target prediction step and obtained a conserved module of 5 genes.

The ability of the obtained target genes to correctly classify soft and stiff cell states is next tested in silico on the validation datasets (Figure 1c) using the area under the curve of the receiver-operator characteristics $(A \cup C-R O C)^{45}$. The best scoring targets are validated experimentally by monitoring mechanical phenotype changes upon their overexpression and downregulation in the cells of choice (Figure 1d). 


\section{Model systems characterized by mechanical phenotype changes}

To curate the datasets, we screened the projects ongoing in our group and identified biological systems for which published transcriptomic data were available, and the concomitant mechanical phenotype changes were either already documented or implicated (Table 1). The mechanical phenotype of the different cell states in each system was characterized using RT-DC. RT-DC is a microfluidic method that relies on flowing cells through a narrow constriction of a microfluidic channel and high-speed imaging to assess the ensuing cell deformation ${ }^{44}$ (Supplementary Figure 1a-b). In the context of this method, the mechanical phenotype is understood as whole-cell elasticity quantified by an apparent Young's modulus, $E$, deduced from cell size and deformation under given experimental conditions $^{46}$ (Supplementary Figure 1c-d). Young's modulus quantifies how much stress (force per unit area) is necessary to deform a cell to a certain extent (i.e., strain), thus higher Young's modulus values indicate that a cell is harder to deform, or stiffer.

The first system included in our analysis encompassed patient-derived glioblastoma cell lines cultured in conditions supporting different levels of activation of the STAT3-Ser/Hes3 signaling axis involved in cancer growth regulation. As previously demonstrated, the higher the STAT3-Ser/Hes3 activation in the characterized states, the stiffer the measured phenotype of glioblastoma cells ${ }^{42}$ (Figure 2a). The second system included small-cell and non-small-cell human carcinoma cell lines originating from intestine, lung, and stomach. Consistently across tissues, small cell-carcinoma cells had a lower apparent Young's modulus compared to their non-small-cell counterparts (Figure 2b). Small-cell carcinomas have relatively small cell sizes, short doubling times and higher metastatic potential connected with poor clinical prognosis in patients ${ }^{47,48}$. In the third studied system, CD34-positive human hematopoietic stem and progenitor cells (HSPCS) isolated from mobilized peripheral blood showed a decrease in the apparent Young's modulus upon treatment with a histone deacetylase inhibitor, valproic acid (VPA), that allows for extensive expansion of HSPCs in vitro $^{49}$ (Figure 2c). In the fourth studied system, two non-tumorigenic breast epithelium MCF10A cell lines bearing single-allele oncogenic mutations in the catalytic subunit alpha of 
the phosphatidylinositol-4,5-bisphosphate 3-kinase (PIK3CA) ${ }^{50}$ showed increased stiffness compared to wild type control cells (Figure 2d). The studied mutations (E545K in exon 9 and H1047R in exon 20) lead to constitutive activation of PIK3CA and an aberrant triggering of the PI3K-AKT-mTOR signaling pathway leading to growth factor-independent proliferation $^{51,52}$. In the fifth system, the fuzzy-colony forming (F-class) state of iPSCs had a lower stiffness as compared to the bone-fide compact-colony forming (C-class) state ${ }^{43}$ (Figure 2e). C-class cells establish endogenous expression of reprogramming factors at moderate levels towards the end of reprogramming, while F-class cells depend on the ectopic expression of the pluripotency factors and are characterized by a fast proliferation rate $^{53}$. Finally, we characterized three stages of developing neurons isolated from embryonic mouse brain ${ }^{54}$, and observed that the stiffness of the cells increased progressively with increasing neurogenic commitment; with differentiating progenitors (DPs) exhibiting a higher apparent Young's modulus than proliferating progenitors (PPs) and newborn neurons (NNs) exhibiting the highest apparent Young's modulus (Figure 2f). Cell areas and deformations used for Young's modulus extraction in all datasets are visualized in

\section{Supplementary Figure 2.}

The six mechano-transcriptomic datasets collected within the framework of our study (Table 1) represent a broad spectrum of biological systems encompassing distinct cell states associated with mechanical phenotype changes. The included systems come from two different species (human and mouse), several tissues (brain, intestine, lung, stomach, bone marrow, breast, as well as embryonic tissue) and are associated with processes ranging from cancerogenic transformations to cell morphogenesis. This high diversity is important for focusing the analysis on genes universally connected to the change in mechanical properties, rather than on genes specific for processes captured by individual datasets. 
a

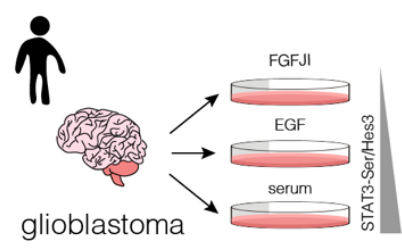

C

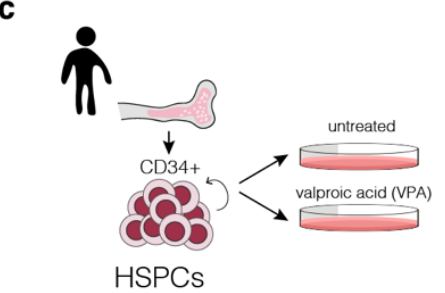

e

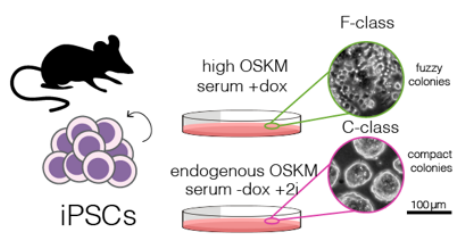

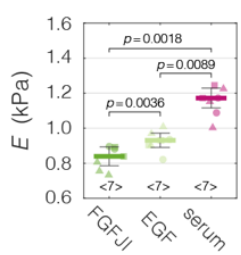
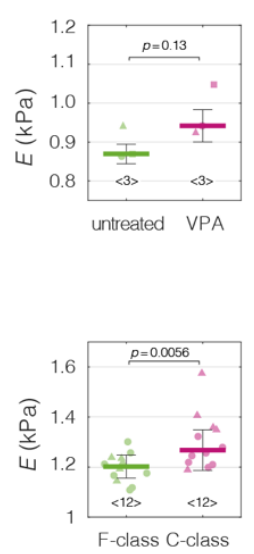

b
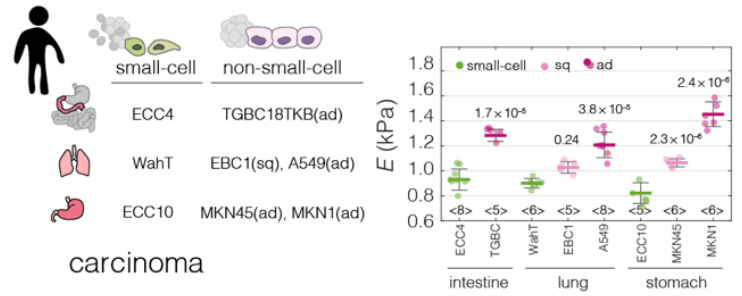

d
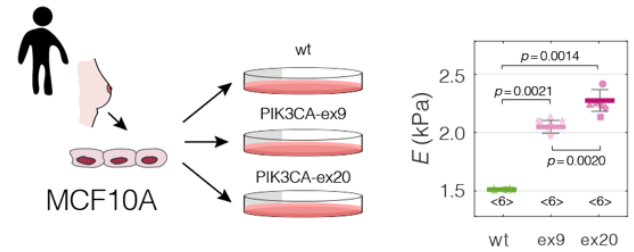

$\mathbf{f}$

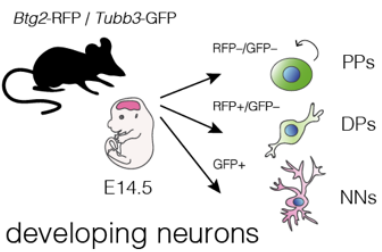

Figure 2| Mechanical properties of divergent cell states in six biological systems. Schematic overviews of the systems used in the mechanomics study, alongside with the cell stiffness of individual cell states parametrized by Young's moduli E. a, Human patient-derived glioblastoma cells with three distinct signaling states maintained by indicated culture conditions. b, Small-cell and two non-smallcell human carcinoma cell types (squamous cell carcinoma, sq, and adenocarcinoma, ad) originating from intestine, lung, and stomach. c, CD34-positive HSPCs mobilized from human bone marrow treated with valproic acid (VPA) or PBS as control. d, Human breast epithelium MCF10A cell lines bearing single-allele mutation in the PIK3CA gene in the exon 9 (E545K) or exon 20 (H1047R), together with parental wild type (wt) as a control. e, F- and C-class murine iPSCs cultured in the presence or absence of doxycycline (dox) activating ectopic expression of OSKM factors (Oct4, Sox2, KIf4, and cMyc). f, Developing neurons isolated from murine embryonic brains at three stages of neural commitment: proliferating progenitors (PPs), differentiating progenitors (DPs) and newborn neurons (NNs). Horizontal lines delineate medians with mean absolute deviation (MAD) as error, datapoints represent medians of the individual replicates, the number of independent biological replicates is indicated below each box. Statistical analysis was performed using generalized linear mixed effects model. Data in a and e were previously published in ref ${ }^{42}$ and ${ }^{43}$, respectively. 
Table 1| Mechano-transcriptomic datasets used in this study. P - prediction, V - validation, HT Seq - high-throughput RNA sequencing, CAGE - cap analysis gene expression, AFM - atomic force microscopy, sq - squamous cell carcinoma, adeno - adenocarcinoma, wt - wild type, PP - proliferating progenitors, DP - differentiating progenitors, NNs - newborn neurons.

\begin{tabular}{|c|c|c|c|c|c|c|c|c|c|c|}
\hline \multicolumn{4}{|c|}{ general information } & \multicolumn{5}{|c|}{ transcriptomic data } & \multicolumn{2}{|c|}{ mechanics data } \\
\hline source & dataset name & $\begin{array}{l}\text { used } \\
\text { for }\end{array}$ & cell states & $\begin{array}{l}\text { accession } \\
\text { number }\end{array}$ & reference & technology & $\begin{array}{l}\text { unique } \\
\text { entries }\end{array}$ & $\begin{array}{c}\text { total } \\
\text { samples }\end{array}$ & method & reference \\
\hline \multirow{4}{*}{$\begin{array}{l}\frac{1}{\sqrt{0}} \\
\stackrel{g}{5} \\
\underline{c}\end{array}$} & glioblastoma & $P$ & FGFJI | EGF | serum & $\begin{array}{c}\text { GEO: } \\
\text { GSE77751 }\end{array}$ & Poser et al. ${ }^{42}$ & HT seq & 39400 & 27 & RT-DC & Poser et al. ${ }^{42}$ \\
\hline & carcinoma & V & small-cell | sq | adeno* & $\begin{array}{c}\text { DDBJ: } \\
\text { DRA000991 }\end{array}$ & $\begin{array}{c}\text { FANTOM } \\
\text { consorptium }^{55}\end{array}$ & CAGE & 18821 & 8 & RT-DC | AFM & this paper \\
\hline & HSPCS & V & untreated | VPA-treated & $\begin{array}{c}\text { GEO: } \\
\text { GSE90552 }\end{array}$ & $\begin{array}{c}\text { Arulmozhivarman } \\
\text { et al. }{ }^{49}\end{array}$ & HT seq & 40101 & 6 & RT-DC & this paper \\
\hline & MCF10A & V & wt | PIK3CA mutation & $\begin{array}{c}\text { GEO: } \\
\text { GSE69822 }\end{array}$ & Kiselev et al. ${ }^{56}$ & HT seq & 38508 & 6 & RT-DC & this paper \\
\hline \multirow{2}{*}{ 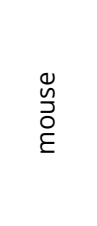 } & iPSCs & $P$ & F-class | C-class & $\begin{array}{c}\text { GEO: } \\
\text { GSE49940 }\end{array}$ & Tonge et al. ${ }^{53}$ & microarray & 18118 & 28 & RT-DC | AFM & Urbanska et al..$^{43}$ \\
\hline & $\begin{array}{l}\text { developing } \\
\text { neurons }\end{array}$ & v & PPs | DPs | NNs & $\begin{array}{c}\text { GEO: } \\
\text { GSE51606 }\end{array}$ & Aprea et al. ${ }^{54}$ & HT seq & 21110 & 9 & RT-DC & this paper \\
\hline
\end{tabular}

*from 3 tissues: intestine, lung, stomach

sthe data for samples of interest was extracted using TET tool from the FANTOM5 website https://fantom.gsc.riken.jp/5/ 


\section{Discriminative network analysis on discovery datasets}

After characterizing the mechanical phenotype of the cell states, we set out to use the accompanying transcriptomic datasets to elucidate genes associated with the mechanical phenotype changes across the different model systems. To this end, we applied a method for inferring phenotype-associated functional network modules from omics datasets termed PC-Corr ${ }^{41}$ on two discovery datasets, and overlayed the results to obtain a conserved module of genes. Thanks to the combination of Pearson's correlation and the discriminative information included in the PC loadings, the PC-corr analysis does not only consider gene co-expression - as is the case for classical co-expression network analysis ${ }^{57,58}$ - but also incorporates the relative relevance of each feature for discriminating between two or more conditions - in our case, the conditions representing soft and stiff mechanical phenotypes.

For the network construction, we chose two datasets that originate from different species, concern unrelated biological processes, and have the highest number of samples included in the transcriptional analysis: human glioblastoma and murine iPSCS (Table 1). PC-corr analysis was performed on these discovery datasets individually using a subset of transcripts at which the two datasets intersect (Figure 3a). First, the 9,452 unique genes from the intersection were used to perform principal component analysis (PCA) (Figure 3b-c). Next, the PC loadings for the component showing good separation between the different cell states (PC1 for both of presented datasets) were normalized and scaled. The processed PC loadings, $V$, were then combined with Pearson's correlation coefficients, $c$, to obtain a $P C$-corr value for each pair of genes $i, j$ for every $n$-th dataset according to the following formula:

$$
P C-\operatorname{corr}_{i, j}^{n}=\operatorname{sgn}\left(c_{i, j}^{n}\right) \min \left(\left|V_{i}^{n}\right|,\left|V_{j}^{n}\right|,\left|c_{i, j}^{n}\right|\right)
$$

The sign of the $P C$-corr value corresponds to the correlated or anti-correlated expression of genes $i, j$, and the magnitude of PC-corr conveys the combined information about the strength of the expression correlation and the contribution of the individual genes to the phenotype-based separation of samples. 
To combine the PC-corr results obtained for the discovery datasets, a combined $P C$-corr value, $P C$ - $\operatorname{corr}_{i, j}^{c o m b}$, was calculated either as a mean or as a minimum of the individual values. For $n$ datasets:

$$
P C-\operatorname{corr}_{i, j}^{c o m b}=\left\{\begin{array}{l}
\delta_{i, j} \frac{1}{N} \sum_{n=1}^{N}\left|P C-\operatorname{corr}_{i, j}^{n}\right| \\
\delta_{i, j} \min \left(\left|P C-\operatorname{corr}_{i, j}^{1}\right|, \ldots,\left|P C-\operatorname{corr}_{i, j}^{n}\right|\right)^{\prime}
\end{array}\right.
$$

where $\delta_{i, j} \in\{-1,1\}$ defines the sign of $P C$ - $\operatorname{corr}_{i, j}^{c o m b}$, and is equal to the mode of $P C$ - $\operatorname{corr}_{i, j}$ signs over all individual datasets. In our implementation on two datasets, gene pairs with opposing PC-corr signs were masked by setting their PC-corr ${ }^{\text {comb }}$ values to zero.

To obtain the network of putative target genes, a cut-off was applied to the absolute value of $P C$-corr ${ }^{c o m b}$. We explored several cut-off strategies in order to obtain a wide overview of

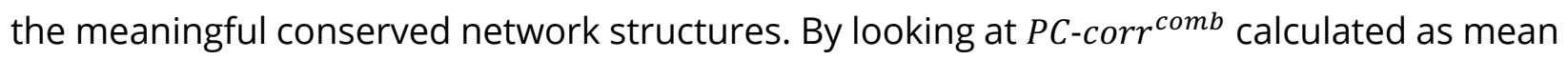
and setting the threshold for its absolute value to 0.75 , we obtained a network of 29 nodes connected by 30 edges (Figure 3e). The edges describe the connection between the genes in the network and their thickness is defined by the $P C$-corr ${ }^{\text {comb }}$ values (Supplementary Table 1). The node colors reflect the strength of the contribution of individual genes to the separation of the different classes as described by the mean of the processed PC loadings $V$.

The obtained network can be made more conservative by using the minimum $P C$-corr ${ }^{\text {comb }}$ instead of the mean, or by changing the cut-off value. Utilizing the $P C$-corr ${ }^{\text {comb }}$ calculated as minimum value (Supplementary Table 2) and setting the cut-off value to 0.70, we obtained a network with 22 nodes connected by 29 edges (Figure 3f). Increasing the cut-off value to 0.75 resulted in a network of 9 genes connected by 12 edges (Figure $\mathbf{3 g}$ ). The list of genes from the three networks presented in Figure $\mathbf{3 e - g}$, together with their full names and processed PC loading values, is presented in Supplementary Table 3.

Finally, we performed gene ontology enrichment analysis for biological processes on the nodes of the network presented in Figure $\mathbf{3 g}$, as well as the union of all nodes presented in Figures 3e-g (Supplementary Figure 3). The top two significantly enriched terms in the 9gene set were the negative regulation of transcription by polymerase II (GO:000122) and 
negative regulation of endothelial cell proliferation (GO:0001937). In the 34-gene set, apart from a broad term of signal transduction (GO:0007165), the significantly enriched terms included negative regulation of transcription by polymerase II (GO:000122), regulation of cell growth (GO:0001558), and negative regulation of cell proliferation (GO:0008285), among others. The aforementioned categories included mostly genes showing high expression in the stiff states. Hence, these results point towards reduced transcriptional activity and reduced growth/proliferation in stiff compared to soft cells.

\section{The identified conserved functional network module comprises five genes}

Regardless of the strategy chosen for the selection of the network-building gene pairs, a strongly interconnected module of 5 genes (Table 1), highlighted in yellow in Figure 3e-g, emerged. We focused on the five genes from this conserved network module as putative targets for regulating cell mechanics: CAV1, FHL2, IGFBP7, TAGLN, and THBS1. Caveolin-1, CAV1, is a protein most prominently known for its role as a structural component of caveolae. Caveolae are small cup-shaped invaginations in the cell membrane that are involved, among other functions, in the mechanoprotective mechanism of buffering the plasma membrane tension ${ }^{59-61}$. Apart from membrane organization and membrane domain scaffolding, CAV1 plays a role in an array of non-caveolar functions such as metabolic regulation or Rhosignalling 59,62,63. The second identified target, four and a half LIM domains 2, FHL2, is a multifaceted LIM domain protein with many binding partners and a transcription factor activity $^{64}$. FHL2 has recently been shown to remain bound to actin filaments under high tension, and be shuttled to the nucleus under low cytoskeletal tension ${ }^{65,66}$ - a property conserved among many LIM domain-containing proteins ${ }^{66,67}$. The third target, Insulin-like growth factor binding protein 7, IGFBP7, is a secreted protein implicated in a variety of cancers. It is involved in the regulation of processes such as cell proliferation, adhesion, and senescence $^{68}$. Transgelin, TGLN, is an actin-binding protein whose expression is up-regulated by high cytoskeletal tension ${ }^{69}$ and is also known to play a role in cancer ${ }^{70}$. Finally, thrombospondin 1, THBS1, is a matricellular, calcium-binding glycoprotein that mediates cell-cell and cell-matrix adhesions and has many regulatory functions ${ }^{71,72}$. 


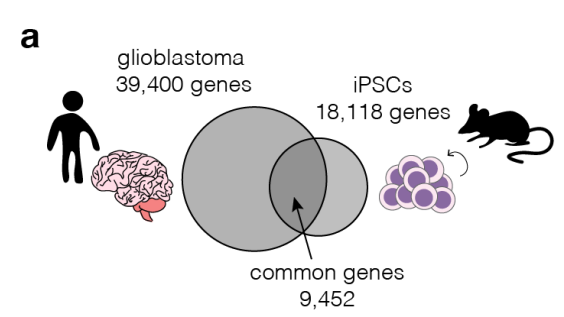

d
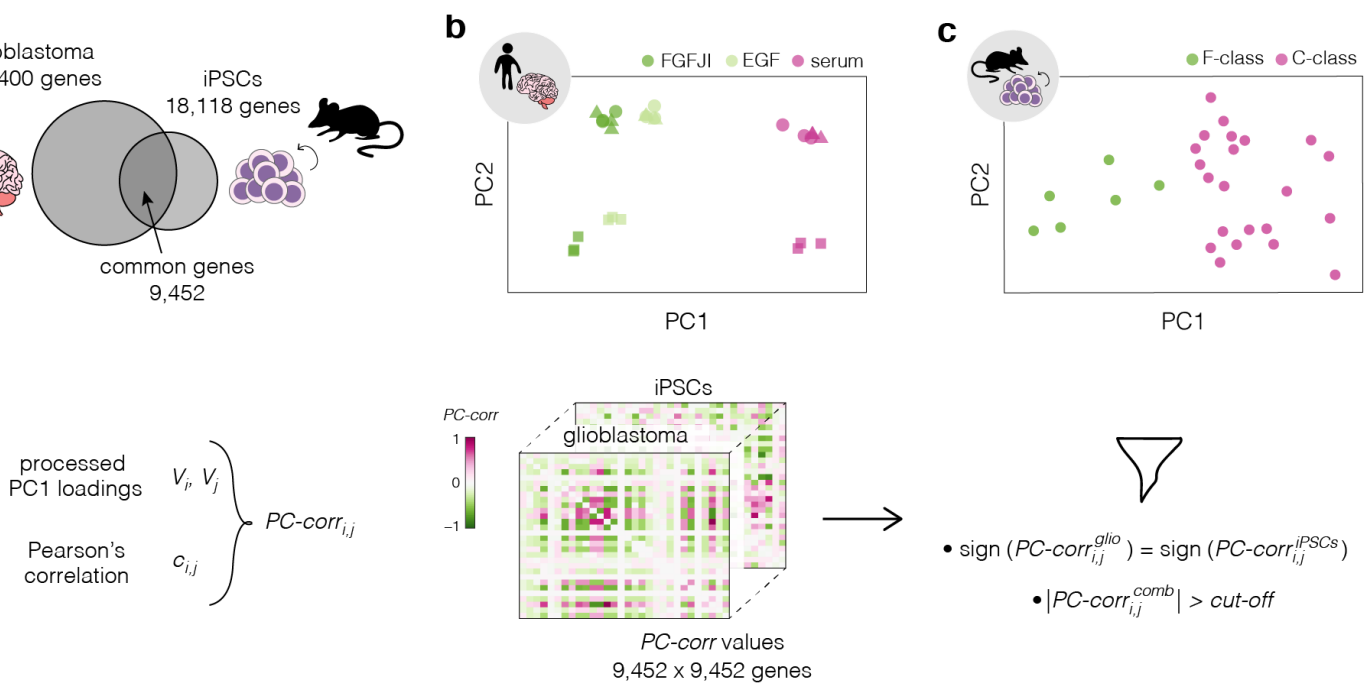

e
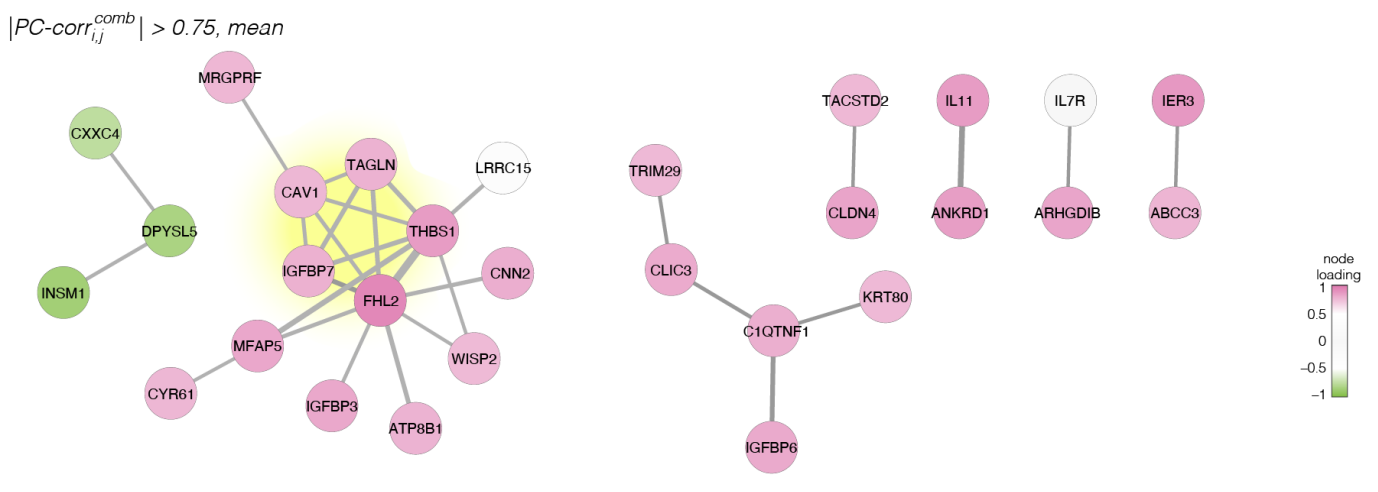

$\mathbf{f}$

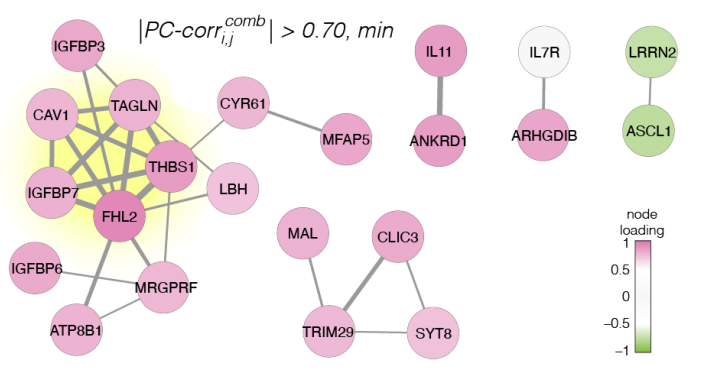

$\mathbf{g}$

$\mid P C$-corr comb $_{i, j} \mid>0.75$, min
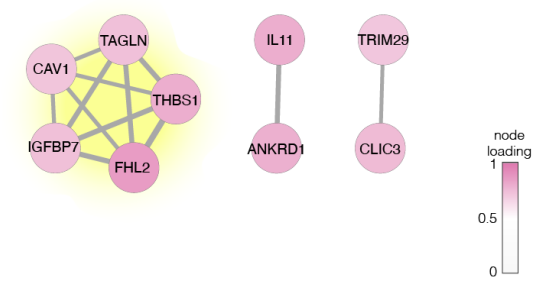

Figure 3| Identification of putative targets involved in cell mechanics regulation.

a, Glioblastoma and iPSC transcriptomes used for the target prediction intersect at 9,452 genes. bc, PCA separation along two first principal components of the mechanically distinct cell states in the glioblastoma (b) and iPSC (c) datasets. The analysis was performed using the gene expression data from the intersection presented in (a). d, Schematic representation of PC-corr analysis and the combination of the PC-corr results for two systems. $\mathbf{e}-\mathbf{g}$, Gene networks based on filtering gene pairs by the combined $P C$-corr score. The presented networks were obtained by setting the cut-off value to 0.75 , when using the mean PC-corr approach (e), and to 0.70 ( $f$ ) and 0.75 (g), when using the minimum value approach. In e-g edge thicknesses represent the $\mid P C$-corr ${ }^{\text {comb }} \mid$ and the colors of the nodes represent the average processed PC loadings. 
Table 2| List of identified target genes comprising the conserved module.

\begin{tabular}{llll}
\hline symbol & gene description & HGNC ID & MGI ID \\
\hline CAV1 & caveolin 1 & HGNC:1527 & MGl:102709 \\
FHL2 & four and a half LIM domains 2 & HGNC:3703 & MGl:1338762 \\
IGFBP7 & insulin like growth factor binding protein 7 & HGNC:5476 & MGl:1352480 \\
TAGLN & transgelin & HGNC:11553 & MGl:106012 \\
THBS1 & thrombospondin 1 & HGNC:11785 & MGl:98737
\end{tabular}

Before validating the performance of the five target genes, we inspected their expression across the divergent cell states in the collected datasets. The target genes show clear differences in expression levels between the soft and stiff cell states and provide for fairly good clustering of the samples corresponding to different cell stiffnesses in both discovery and validation datasets (Supplementary Figure 4). The relation between normalized apparent Young's modulus change and fold-change in the expression of the target genes is presented in Supplementary Figure 5. Of note, the direction of changes in the expression levels between the soft and stiff cell states in the validation datasets was not always following the same direction (Supplementary Figure 4c-f, Supplementary Figure 5). This suggests that the genes involved in cell mechanics regulation may not have a monotonic relationship with cell stiffness, but rather are characterized by different expression regimes in which the expression change in opposite directions can have the same effect on cell stiffness.

\section{CAV1 performs best at classifying soft and stiff cell states in validation datasets}

Next, we used the four remaining datasets (carcinoma, HSPCs, MCF10A and developing neurons) to computationally test the performance of the five identified genes in classifying the individual samples into soft or stiff phenotypes based on their transcription levels. For this purpose, we implemented the AUC-ROC analysis ${ }^{45}$. The ROC curve is a graphical plot that illustrates the classification ability of a binary classifier system. On the $x$ axis of an ROC plot, the false positive rate (FPR) is represented, and on the $y$ axis the true positive rate (TPR). We built ROC curves for every soft-stiff pair of cell states from individual datasets by swiping through different thresholds of the expression of a given gene and calculating the TPR and 
FPR for classifying the soft and stiff cell states based on these thresholds (Supplementary Figure 6). We then used the area under the ROC curve as a proxy for the performance of these one-feature classifiers. AUC-ROC takes values from 0 to 1 , with 1 corresponding to a perfect classifier and 0.5 to a random classifier. The AUC-ROC values obtained for each gene from the conserved module in the respective validation datasets are summarized in Table 3. We found that CAV1, with an average AUC-ROC score of 0.95, was the best performing classifier. Thus, we set out to test experimentally if modifying the levels of CAV1 in cells could elicit the predicted change in their mechanical phenotype.

Table 3 | In silico validation of the genes from the conserved functional network module using AUC-ROC. The table contains values of AUC-ROC for target genes obtained for every soft-stiff cell state pair. The average of AUC-ROC values obtained for all validation systems is included in the last table row. sc - small cell carcinoma, sq - squamous cell carcinoma, adeno - adenocarcinoma, wt, wild type, cnt - untreated control, VPA - valproic acid, PPs - proliferating progenitors, DPs - differentiating progenitors, NNs - newborn neurons.

\begin{tabular}{ccccccc}
\hline dataset & state & CAV1 & FHL2 & IGFBP7 & TAGLN & THBS1 \\
\hline \multirow{2}{*}{ carcinoma } & sc vs sq & 1 & 0.84 & 0.87 & 0.77 & 1 \\
& sq vs adeno & 0.84 & 0.61 & 0.51 & 0.72 & 0.61 \\
SC vs adeno & 1 & 0.90 & 0.82 & 0.91 & 0.74 \\
MSP10A & wt vs H1047R & 0.78 & 1 & 1 & 1 & 1 \\
HSPCS & cnt vs VPA & 1 & 0.94 & 1 & 0.84 & 0.63 \\
& PPs vs DPs & 1 & 1 & 0.78 & 0.50 & 1 \\
developing neurons & DPs vs NNs & 1 & 1 & 0.78 & 0.67 & 1 \\
& PPs vs NNs & 1 & 1 & 0.89 & 1 & 1 \\
\hline & mean & 0.95 & 0.91 & 0.83 & 0.80 & 0.87
\end{tabular}




\section{Perturbing expression levels of CAV1 changes cells stiffness}

The increase in apparent Young's modulus was accompanied by an increase in CAV1 levels in most of our datasets (Supplementary Figure 5a). Additionally, we observed that mouse embryonic fibroblasts isolated from CAV1 knock out mice (CAV1KO) are softer than the wild type cells (WT) (Supplementary Figure 7). Thus, we hypothesized that artificially decreasing the levels of CAV1 should cause cell softening, and conversely, increasing the level of CAV1 should result in higher cell stiffness (Figure 4a). To test this hypothesis, we perturbed the levels of CAV1 in the cell lines representing two intestine carcinoma types: ECC4, the smallcell carcinoma with a comparably soft phenotype, and TGBC18TKB (TGBC), the adenocarcinoma with a comparatively stiff phenotype. We confirmed that TGBC cells have a higher level of CAV1 as compared to ECC4 on a protein level (Supplementary Figure 8a) and that they are characterized by a stiffer phenotype, not only when measured with RT-DC (Figure 1b), but also with atomic force microscopy (AFM) using both standard indentation experiments, as well as oscillatory indentation at different frequencies, referred to as AFM microrheology (Supplementary Figure 8b-c).

To decrease the levels of CAV1 in the TGBC cells, we performed knock-down experiments using two RNA interference (RNAi) systems, endoribonuclease-prepared siRNA (esiRNA) targeting three different parts of CAV1 transcript (esiCAV1-1, esiCAV1-2, and esiCAV1-3), and a pool of conventional siRNAs (CAV1-pool). All the RNAi approaches resulted in the decrease of the apparent Young's modulus of TGBC cells as measured by RT-DC (Figure $\mathbf{4 b - c}$, Supplementary Figure 9a-b), the most prominent effect was observed using esiCAV1-1. We further confirmed that CAV1 knock-down with esiCAV1-1 resulted in decreased stiffness of TGBC cells using AFM indentation and AFM microrheology measurements (Supplementary Figure 10).

To investigate the influence of increased CAV1 levels on cell stiffness, we performed transient overexpression experiments of CAV1 with a dTomato reporter under independent ribosomal entry site, IRES, (CAV1 iT) in both ECC4 and TGBC cell lines. At 72 hours post transfection, we observed elevated levels of CAV1 in both cell lines on a protein level in bulk (Figure 4d). Since 
in the transient overexpression experiments not all of the cells are transfected, we leveraged the possibility to monitor the fluorescence of single cells in parallel with their mechanical phenotype offered by real-time fluorescence and deformability cytometry (RT-FDC) ${ }^{73}$ to gate for the fluorescence-positive cells $(T+$, gate marked in magenta in Figure 4e). The fluorescence-positive cells in the CAV1-transfected sample, CAV1iT+, showed higher apparent Young's moduli as compared to fluorescence-negative cells in both control sample (mock) and CAV1-transfected sample (CAV1iT-, internal control) (Figure 4f, Supplementary Figure 9c-d). The effect was observed in ECC4 as well as TGBC cells, however, it was more pronounced in the TGBC cells, suggesting that the cells may be more responsive to the artificial increase in CAV1 levels when natively expressing a basal level of this protein.

Finally, we performed CAV1 perturbation experiments in a breast epithelial cell model of cancerous transformation, MCF10A-ER-Src cells, in which the Src proto-oncogene can be induced by treatment with tamoxifen (TAM). As previously shown, TAM addition triggers Src phosphorylation and cellular transformation ${ }^{74}$, which is associated with F-actin cytoskeletal changes and, after a transient stiffening, the acquisition of a soft phenotype evident at 36 hours post induction ${ }^{75}$. We inspected a previously published microarray dataset and determined that the expression of CAV1 diminishes over time after TAM treatment ${ }^{76}$ (Supplementary Figure 11a). We then showed that the decrease of CAV1 could be observed on protein level at 72 hours post induction (Supplementary Figure 11b), a timepoint at which the TAM-induced MCF10A-ER-Src cells show a significant decrease in cell stiffness (ref ${ }^{75}$ and Supplementary Figure 11c). We next showed that decreasing the level of CAV1 by knock-down caused a decrease in stiffness of uninduced MCF10A-ER-Src cells similar to that caused by TAM induction (Supplementary Figure 11d). We then performed an inverse experiment, in which we rescued the CAV1 levels in TAM-induced MCF10A-ER-Src cells by transient overexpression. The cells with CAV1 overexpression showed a stiff phenotype, similar to the one of uninduced cells (Supplementary Figure 11e). 
Taken together, the results obtained with the intestine carcinoma cell lines and MCF10A-ER-Src cells show that CAV1 not only correlates with, but also is causative of mechanical phenotype change.

a

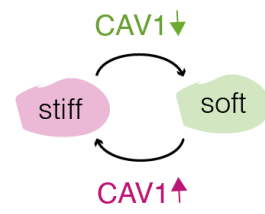

d

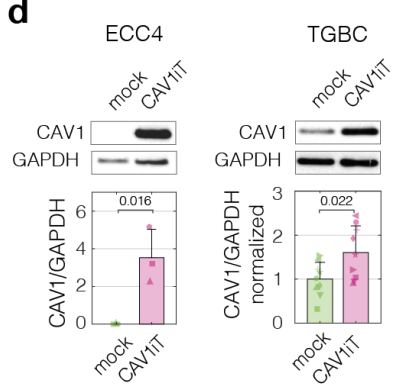

b

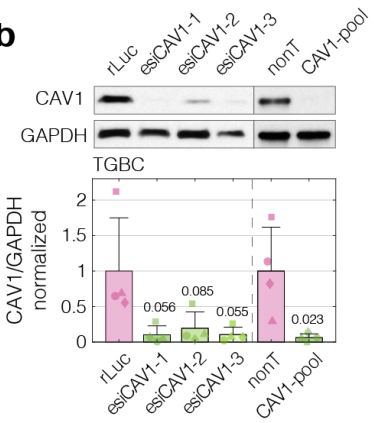

e
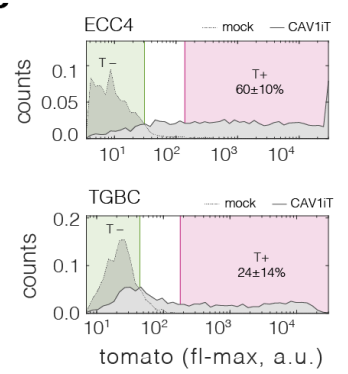

C

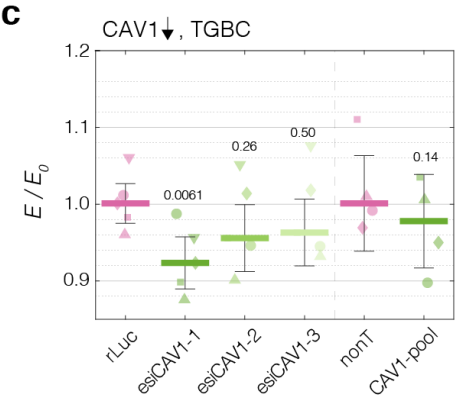

$\mathbf{f}$

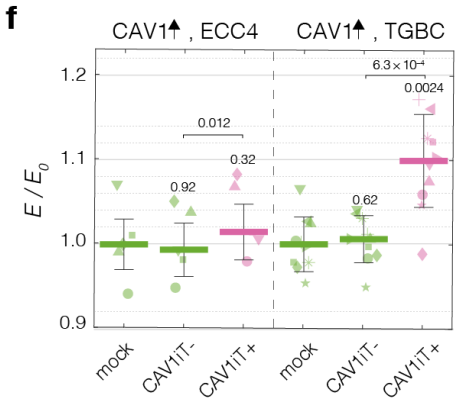

Figure 4| Perturbing levels of CAV1 affects the mechanical phenotype of intestine carcinoma cells. a, Putative relationship between the CAV1 levels and the mechanical phenotype. b, Verification of CAV1 knock-down in TGBC cells using Western blot analysis, representative blot (top) as well as quantification (bottom, $n=4$ ) are shown. c, Apparent Young's modulus of TGBC cells upon CAV1 knock-down as measured by RT-DC, normalized to respective non-targeting controls $(n=5$ and 4 for set 1 and 2, respectively). d, Verification of CAV1 overexpression in ECC4 and TGBC cells using WB analysis, representative blot (top) as well as quantification (bottom, $n=3$ and 9, for ECC4 and TGBC, respectively) are shown. e, Gating for fluorescence positive and negative cells based on dTomato expression in ECC4 (top) and TGBC (bottom) cells. Fluorescence positive cells correspond to cells expressing CAV1-IRES-dTomato. f, Apparent Young's modulus of ECC4 and TGBC cells upon CAV1 overexpression as measured by RT-DC, normalized to mock controls ( $n=5$ and 9 for ECC4 and TGBC, respectively). The bar plots in $\mathbf{b}$ and $\mathbf{d}$ show means with standard deviation as error. In $\mathbf{c}$ and $\mathbf{f}$, horizontal lines delineate medians with mean absolute deviation (MAD) as error, datapoints represent medians of the individual replicates. Statistical analysis was performed using two-sided two-sample $t$-tests in $\mathbf{b}$ and $\mathbf{d}$, and generalized linear mixed effects models in $\mathbf{c}$ and $\mathbf{f}$. 


\section{Discussion}

The mechanical phenotype of cells is recognized as a hallmark of many physiological and pathological processes. Understanding how to control it is a necessary next step that will facilitate exploring the impact of cell mechanics perturbations on cell and tissue function 4 . The increasing availability of transcriptional profiles accompanying cell state changes, has recently been complemented by the ease of screening for mechanical phenotypes of cells thanks to the advent of high-throughput microfluidic methods ${ }^{77}$. This provides an opportunity for data-driven identification of genes involved in the control of mechanical cell phenotype in a hypothesis-free manner. Here we leveraged this opportunity by performing discriminative network analysis on transcriptomes associated with mechanical phenotype changes to elucidate a conserved module of five genes potentially involved in cell mechanical phenotype regulation. We evaluated the prediction performance of individual genes when classifying cells into soft and stiff states in silico, and demonstrated on the example of best performing gene, CAV1, that its experimental up- and downregulation impacts the stiffness of the measured cells. This demonstrates that the level of CAV1 not only correlates with, but also is causative of mechanical phenotype change.

The workflow presented here is a blueprint for data-driven discovery of cell mechanics regulators. Its key features are the hypothesis-free modus operandi and the integration of information from a breadth of biological systems, that allows to focus on genes that play a relatively general role in cell mechanics rather than on genes specific to the individual experimental models. Noteworthy, by including the PC loadings in the scores used for thresholding, the PC-corr method implemented for network analysis in our study offers a multivariate alternative to classical co-expression analysis ${ }^{57,58}$, that highlights not only the correlation between the genes but also their relative importance for separating samples based on their mechanical phenotype. Despite its simplicity, PC-corr offers a robust performance on different types of omics data, and has already proven its efficacy in several studies $^{41,42,78}$. 
Among the target genes elucidated in our analysis, we did not observe enrichment of gene ontology terms related to actin cytoskeleton organization, actomyosin contractility, cell adhesion or cell migration - processes that are typically associated with cell mechanics (Supplementary Figure 3). This can be partially explained by looking at the mRNA rather than the protein level, its supramolecular assembly, activation state or localization. Upon closer inspection of the obtained gene targets individually, we found some links connecting them with cell mechanics in the literature. CAV1, apart from its role in buffering plasma membrane tension ${ }^{60,61}$, has been shown to be involved in cross-talk with Rho-signalling and actin-related processes $59,62,63$, and to correlate with cell stiffness in Ras-transformed fibroblasts $^{79}$. Furthermore, CAV1 was recently shown to modulate the activation of transcriptional cofactor yes-associated protein, YAP, in response to changes in stiffness of cell substrate ${ }^{80}$ and in the mechanical stretch-induced mesothelial to mesenchymal transition ${ }^{81}$. YAP is an established transducer of not only various mechanical stimuli, but also of cell shape and the changes in the actin cytoskeleton tension ${ }^{82}$, the latter being an important determinant of cell stiffness. Conversely, YAP is an essential co-activator of CAV1 expression $^{83}$. In the extended networks (Figure 3e,f), we found three further genes that are identified (CYR61, ANKRD1) $)^{84,85}$ or implicated (THBS1) ${ }^{82}$ as transcriptional targets of YAP. The next target, transgelin, TGLN (also known as $\mathrm{SM} 22 \mathrm{a}$ ) is an actin-binding protein, that stabilizes actin filaments and is positively correlated with cytoskeletal tension ${ }^{86}$. Transgelin is a member of the calponin protein family, one further member of which, calponin 2, CNN2, is present in the broader sets of genes identified in this study (Figure 3e,f, Supplementary Table 3). The expression of calponin 2, likewise, stabilizes actin filaments and is increased in cells with high cytoskeletal tension ${ }^{87,88}$. Finally, FHL2 is a transcriptional coactivator that is found, together with other LIM domain protein families such as zyxin and paxillin, to localize to actin filaments that are under stress ${ }^{65-67}$. When the cytoskeletal tension is low, FHL2 translocates to the nucleus, thus serving as a nuclear transducer of actomyosin contractility $^{65}$. 
As seen from the example of the target genes included in the conserved module, their change is correlated with cell mechanics across all datasets, but it does not always follow the same trend (Supplementary Figure 4 and 5). This non-monotonic relationship between gene expression and the mechanical phenotype change suggests that there may be different regimes at which the expression change in the same direction has an opposite effect on the property of interest. Furthermore, the effect of expression change may be contextual and depend on the state of cells. This observation carries some parallels to the role of several of our target genes in cancer progression. For example, CAV1 has been indicated as both promoting and suppressing cancer progression in a variety of tissues. One way in which this can be reconciled is that the change in CAV1 expression may have different roles depending on the stage of caner progression ${ }^{62,89,90}$. A similar ambiguity of their role in cancer progression was indicated for THBS1 $1^{72}$ and IGFBP7 ${ }^{68}$. Apart from characterizing the response regimes, it will be also important to consider the temporal dynamics of cell response to the change in expression of a given gene. Trying to push the cell out of its equilibrium may cause the system to respond actively to counterbalance the induced change, which, in turn, may lead to oscillations in both expression levels of manipulated protein and its effectors, as well as the mechanical properties of the cell.

Among all different types of omics data, looking at the transcriptome is advantageous and disadvantageous at the same time. Its limitation is that mRNA levels do not necessarily reflect protein content in cells. Furthermore, for many proteins it is not the absolute level that has a functional relevance, but rather the protein activation by, for example, phosphorylation or binding with co-activators, or its localization. However, identifying the players at the transcriptome level has the advantage of easy implementation in perturbation experiments with established genetic tools, such as CRISPR-Cas technology or RNAi. Furthermore, the presented analysis framework is readily applicable to other types of omics data, including proteomic, metabolomic, lipidomic, or glycomic data, the analysis of which would complement our study and provide different insights into the regulation of cell mechanics. 
Lipidomic data, for example, could reveal possible contributors to cell mechanics related to the composition of cell membrane.

For the approaches such as the one pioneered in this study to flourish, it is necessary that the mechanical datasets become routinely published and annotated in a manner similar to omics datasets. With the recent advent of high-throughput cell mechanical characterization techniques, such as deformability cytometry methods ${ }^{77}$, the establishment of a database for cell mechanics gains immediate relevance. In our group alone, within the timespan of five years since the RT-DC method was originally published ${ }^{44}$, we have accumulated over 100,000 individual mechanical characterization experiments, comprising roughly a billion of single cells measured. Once a vast number of mechanics datasets connected to omics profiles is available, it will be straightforward to develop a next generation artificial intelligence algorithm predicting cell stiffness from given omics profiles. Apart from analyzing divergent cell states, the search for mechanical regulators could be complemented by looking into omics data of cells from unimodal populations sorted by their mechanical properties $-\mathrm{a}$ pursuit that with the advent of high-throughput methods for mechanics-based sorting of cells, such as sorting RT-DC ${ }^{91}$ or passive filtration-based approaches ${ }^{92}$, becomes a realistic objective.

In conclusion, this work brings together machine learning-driven discriminative network analysis and high-throughput mechanical phenotyping to establish a blueprint workflow for data-driven de novo identification of genes involved in the regulation of cell mechanics. Ultimately, providing the ways to tune the mechanical properties on demand will enable turning cell mechanics from a correlative phenomenological parameter to a controllable property. Such control will, in turn, allow us to interfere with important processes such as tissue morphogenesis, cell migration, or circulation through vasculature. 


\section{Online Methods}

\section{CELL CULTURE}

\section{Glioblastoma cell lines}

The glioblastoma dataset contained three primary human brain tumour cell lines (X01, X04, and $\mathrm{X08}$ ) in three distinct signalling states. The cells were cultured and characterized within a framework of a previous study ${ }^{42}$. In brief, the three signalling states characterized by low, medium, and high activation of STAT3-Ser/Hes3 signalling axis, were maintained by growth media containing fetal bovine serum (serum), epidermal growth factor (EGF), or basic fibroblast growth factor combined with a JAK inhibitor (FGFI), respectively. Upon thawing, cells were expanded in a serum-free DMEM/F12 medium (10-090-CV, Mediatech, Corning, NY, USA) containing N2 supplement and $20 \mathrm{ng} \mathrm{ml}^{-1} \mathrm{EGF}$ (R\&D Systems, MN, USA) at $37^{\circ} \mathrm{C}$ in a 5\% oxygen incubator. Each cell line was then plated into three separate flasks and cultured in the DMEM/F12 medium containing N2 supplement and additional supplementation of either serum (10\%), EGF (20 ng ml$\left.l^{-1}\right)$, or FGFI (20 ng ml-1 , bFGF, R\&D Systems; and $200 \mathrm{nM}$ JAK inhibitor, Calbiochem, Merck Millipore, Germany). Cells were collected for mechanical characterization and RNA sequencing after 5-day exposure to the respective culture conditions ${ }^{42}$.

\section{Carcinoma cell lines}

Small-cell and non-small-cell carcinoma cell lines from intestine, stomach and lung were acquired from RIKEN BioResource Research Center, Japan (see Supplementary Table $\mathbf{4}$ for the list of cell lines and media). Cells were cultured in growth media supplemented with $5 \%$ (TGBC) or 10\% (rest) heat-inactivated fetal bovine serum (10270106, Gibco, ThermoFisher Scientific, MA, USA) and $100 \mathrm{U} \mathrm{ml}^{-1} / 100 \mathrm{\mu g} \mathrm{ml}^{-1}$ penicillin/streptavidin (15140122, Gibco), at $37^{\circ} \mathrm{C}$ and $5 \% \mathrm{CO}_{2}$. Sub-culturing was performed using trypsin (25200072, Gibco). Cells were collected for mechanical characterization at 70\% confluency. The RNAseq data was obtained from FANTOM5 consortium ${ }^{55}$. 


\section{MCF10A cell lines with PIK3CA mutations}

MCF10A cell lines with single-allele PIK3CA mutation E545K in exon 9 (ex9) or H1024R in exon 20 (ex20) were previously generated by homologous recombination by Horizon Discovery LTD, UK ${ }^{50}$ and were kindly provided, together with an isogenic wild type (wt) control, by L.R. Stephens (Babraham Institute, UK). Cells used for mechanical characterization were cultured in DMEM/F12 medium (31330038, Gibco) supplemented with 5\% horse serum (PAA Laboratories), $10 \mathrm{gg} \mathrm{ml}^{-1}$ insulin (19278, Sigma-Aldrich, MO, USA), $0.2 \mu g \mathrm{ml}^{-1}$ hydrocortisone (H0888, Sigma-Aldrich), $0.1 \mu^{-1} \mathrm{ml}^{-1}$ cholera toxin (C8052, Sigma-Aldrich), and $100 \mathrm{U} \mathrm{ml}^{-1} / 100 \mu \mathrm{g} \mathrm{ml}^{-1}$ penicillin/streptomycin (15140122, Gibco). The wt cells were additionally supplemented with $10 \mathrm{ng} \mathrm{ml}^{-1}$ EGF (E9644, Sigma-Aldrich), while mutant cell liens were maintained without EGF. Sub-confluent cells were collected for mechanical characterization using trypsin (25200056, Gibco). Mechanical data were collected from two biological replicates with three technical repetitions each. The RNAseq data for ex20 and wt cells were retrieved from a previous study ${ }^{56}$, in which cells were cultured in a reduced medium (DMEM/F12 supplemented with 1\% charcoal dextran treated fetal bovine serum, $0.2 \mu \mathrm{g} \mathrm{ml}{ }^{-1}$ hydrocortisone and $0.1 \mu \mathrm{g} \mathrm{ml}^{-1}$ cholera toxin).

\section{$\mathrm{CD}^{+} 4^{+}$hematopoietic stem and progenitor cells}

For isolation of hematopoietic stem and progenitor cells, leukapheresis samples were obtained from G-CSF (granulocyte colony-stimulating factor) mobilized peripheral blood of healthy donors after informed consent (ethical approval no. EK221102004, EK47022007). $\mathrm{CD}^{+}{ }^{+}$cells were isolated via magnetic-activated cell sorting (MACS) and cultured ex vivo as described in detail elsewhere ${ }^{93} .1 \mathrm{mM}$ VPA, or PBS as a control, were administered to CD34 cells after $24 \mathrm{~h}$ in culture. Cells were collected for mechanical characterization and RNA sequencing after 5-day exposure.

\section{Induced pluripotent stem cells}

F- and C-class iPSCs were derived through reprogramming of murine fetal neural progenitor cells with Tet-On system for doxycycline-inducible expression of OSKM (Oct4, Sox2, KIf4, 
CMyc) factors in a previous study ${ }^{43}$. Both iPSCs classes were cultured on $0.1 \%$ gelatin-coated dishes in FCS/LIF medium (DMEM+Glutamax (61965059, Gibco), 15\% fetal calf serum (Pansera ES, PAN-Biotech, Germany), $100 \mu \mathrm{M} \beta$-mercaptoethanol (PAN-Biotech), $2 \mathrm{mM}$ L-glutamine, $1 \mathrm{mM}$ sodium pyruvate, $1 \times$ nonessential amino acids, $15 \mathrm{ng} \mathrm{ml}^{-1}$ recombinant LIF (MPI-CBG, Dresden, Germany)). The F-class iPSCs were additionally supplemented with $1 \mu \mathrm{g} \mathrm{ml}^{-1}$ doxycycline, and the C-class iPSCs with a mixture of two inhibitors (2i): $1 \mu \mathrm{M}$ MEK inhibitor (PD0325901, Calbiochem) and 3 MM GSK3 inhibitor (CH99021, Calbiochem). Cells were passaged and harvested using $0.1 \%$ trypsin solution. The mechanical characterization was performed not earlier than at the $27^{\text {th }}$ day of reprogramming ${ }^{43}$. The microarray expression profiles were retrieved from a previous study, in which the F- and C-class iPSCs were derived from embryonic fibroblasts using similar doxycycline-inducible OSKM expression system ${ }^{53}$.

\section{Developing Neurons}

For isolation of neurons at different developmental stages, we used a double-reporter mouse line $B \operatorname{tg} 2^{R F P} / T u b b 3^{G F P}$. This line enables discrimination of proliferating progenitors (RFP-/GFP-), differentiating progenitors (RFP+/GFP-), and newborn neurons (RFP+/GFP+). Lateral cortices dissected from E14.5 murine embryos were dissociated using a papain-based neural dissociation kit (Miltenyi Biotech, Germany) and the cell populations of interest were separated based on the RFP/GFP expression using FACS as described in detail elsewhere ${ }^{54}$. The three types of sorted cells were then subjected to RNA sequencing ${ }^{54}$ and mechanical characterization.

\section{Mouse embryonic fibroblasts}

Previously established, immortalized WT and CAV1KO mouse embryonic fibroblasts derived from WT and CAV1KO littermate C57BL/9 mice ${ }^{94}$ were used in this study. Cells were cultured in DMEM medium (11960044, Gibco), supplemented with 10\% fetal bovine serum (10270106, Gibco), $2 \mathrm{mM}$ glutamine (25030081, Gibco), $100 \mathrm{U} \mathrm{ml}^{-1} / 100 \mu \mathrm{g} \mathrm{ml}^{-1}$ penicillin/streptomycin 
(15070063, Gibco), at $37^{\circ} \mathrm{C}$ and $5 \% \mathrm{CO}_{2}$. Sub-confluent cells were collected for mechanical measurements by trypsinization (25200056, Gibco).

\section{MCF10A ER-Src cell line}

The MCF10A ER-Src cells were a kind gift from K. Struhl (Harvard Medical School, MA, USA). ER-Src is a fusion of the v-Src (viral non-receptor tyrosine kinase) with the ligand-binding domain of the estrogen receptor, that can be induced by cell treatment with tamoxifen $(\mathrm{TAM})^{74}$. Cells were grown at $37^{\circ} \mathrm{C}$ under $5 \% \mathrm{CO}_{2}$ in DMEM/F12 medium (11039047, Gibco), supplemented with 5\% charcoal (C6241, Sigma-Aldrich)-stripped horse serum (16050122, Gibco), $20 \mathrm{ng} \mathrm{ml}^{-1}$ EGF (AF-100-15, Peprotech), $10 \mathrm{mg} \mathrm{ml}^{-1}$ insulin (I9278, Sigma-Aldrich), 0.5 $\mathrm{mg} \mathrm{ml}^{-1}$ hydrocortisone (H0888, Sigma-Aldrich), $100 \mathrm{ng} \mathrm{ml}^{-1}$ cholera toxin (C8052, SigmaAldrich), and $100 \mathrm{U} \mathrm{ml}^{-1} / 100 \mu \mathrm{g} \mathrm{ml}^{-1}$ penicillin/streptomycin (15070063, Gibco). To induce the Src expression cells were plated at 50\% confluency, and after allowing to adhere for $24 \mathrm{~h}$, treated with $1 \mu \mathrm{M} 4 \mathrm{OH}-\mathrm{TAM}$ (H7904, Sigma-Aldrich) or with identical volume of ethanol as a control. Cells were characterized in adherent state using AFM at timepoints specified in the text.

\section{MECHANICAL MEASUREMENTS}

\section{Mechanical characterization of cells using RT-DC}

RT-DC measurements for mechanical characterization of cells were performed at room temperature according to previously established procedures ${ }^{95}$. In brief, cells were harvested by trypsinization (adherent cells) and/or centrifugation at $400 \mathrm{~g}$ for 3-5 min, and suspended in a measurement buffer (MB). MB (osmolarity $310-315 \mathrm{mOsm} \mathrm{kg}^{-1}, \mathrm{pH} 7.4$ ) was based on phosphate buffered saline without $\mathrm{Mg}^{2+}$ and $\mathrm{Ca}^{2+}$ and contained $0.5 \%$ or $0.6 \%(\mathrm{w} / \mathrm{w}$ ) methylcellulose (4000 cPs, Alfa Aesar, Germany) for increased viscosity. Cells were introduced into a microfluidic chip using a syringe pump (NemeSys, Cetoni, Germany), and focused into a 300- $\mu \mathrm{m}$ long channel constriction (with a square cross-section of $20 \times 20$ or $30 \times 30 \mu \mathrm{m}$ ) by sheath flow infused at a flow rate three times as high as that of the cell suspension. The imaging was performed at the end of the channel constriction 
(Supplementary Figure 1b) at 2,000 frames $\mathrm{s}^{-1}$. The cell area and deformation were derived from the fitted cell contours in real-time by the acquisition software (Shapeln2; Zellmechanik Dresden, Germany). Apparent Young's modulus values were assigned to each cell based on its area and deformation under given experimental conditions (flow rate, channel size, viscosity of the medium, temperature) using a look-up table obtained through numerical simulations of an elastic solid ${ }^{46}$ with the aid of ShapeOut (ShapeOut 1.0.1; https://github.com/ZELLMECHANIK-DRESDEN/ShapeOut; Zellmechanik Dresden). The events were filtered for area ratio (the ratio between the area enclosed by the convex hull of the cell contour and the raw area enclosed by the contour) to discard incomplete contours or cells with rough surface, and for cell area and aspect ratio to discard derbies and doublets. Experimental details (channel sizes, flow rates, measurement buffers) and gates used for filtration in respective datasets are listed in Supplementary Table 5.

\section{Mechanical characterization of cells using AFM}

For AFM measurements, cells were seeded on glass bottom dishes (FluoroDish; FD35100, WPI, FL, USA) at least one day in advance. Mechanical characterization was performed on adherent cells in a sub-confluent culture in $\mathrm{CO}_{2}$-independent medium $(18045054, \mathrm{Gibco})$ at $37^{\circ} \mathrm{C}$ (temperature was maintained by a petri dish heater, JPK Instruments, Germany). AFM measurements on TGBC and ECC4 cell lines were conducted on a Nanowizard 4 JPK Instruments). Tip-less cantilevers (PNP-TR-TL, nominal spring constant $k=0.08 \mathrm{~N} \mathrm{~m}^{-1}$, Nanoworld, Switzerland) decorated a polystyrene bead of 5- $\mu \mathrm{m}$ diameter (PS-R-5.0, microParticles, Germany) each were used as the indenters. The cantilever spring constants were measured prior to each experiment using the thermal noise method implemented in the JPK SPM software (JPK Instruments). For each cell three indentation curves were recorded with a piezo extension speed of $5 \mu^{-1}$ to a maximum set force of $2 \mathrm{nN}$. For the microrheology analysis, the cantilever was lowered using a piezo extension speed of $5 \mu \mathrm{m} \mathrm{s}^{-1}$ until a force set point of $1 \mathrm{nN}$ was reached, corresponding to an approximate indentation depth $\delta_{0}$ of $1 \mu \mathrm{m}$. The lowered cantilever was then oscillated by a sinusoidal motion of the piezo elements at an amplitude of $10 \mathrm{~nm}$ for a period of 10 cycles. The oscillations were 
performed sequentially at different frequencies in the range of $3-200 \mathrm{~Hz}$. Indentation experiments on MCF10A ER-Src cells were conducted as described above, except different tip-less cantilevers (Arrow TL1, nominal spring constant $k=0.35-0.45 \mathrm{~N} \mathrm{~m}^{-1}$, Nanoworld) with a $5-\mu \mathrm{m}$ bead glued at the end were used as the indenter.

\section{AFM indentation data analysis}

Recorded force-distance curves were converted into force-indentation curves and fitted in JPK data processing software (JPK DP, JPK Instruments) using Sneddon's modification of the Hertz model for a spherical indenter ${ }^{96}$ :

$$
F=\frac{E}{1-v^{2}}\left(\frac{a^{2}+r^{2}}{2} \ln \frac{r+a}{r-a}-a r\right)
$$

with

$$
\delta=\frac{a}{2} \ln \frac{r+a}{r-a}
$$

where $F$ denotes the indentation force, $E$ the elastic modulus, $v$ the Poisson's ratio, $a$ the radius of the projected contact area formed between the sample and the indenter, $r$ the radius of the indenter, and $\delta$ the indentation depth. Poisson ratio was set to 0.5 .

\section{AFM microrheology data analysis}

The force and indentation signals from oscillatory measurements were fitted using a sinusoidal function to extract the amplitude and phase angle of each signal. Data were analyzed analogously to the procedure described by Alcaraz et al. ${ }^{97}$ but for a spherical not a pyramidal indenter. Briefly, the method relies on the linearization of the Hertz model for a spherical indenter due to small oscillations by using the first term of the Taylor expansion and subsequent transformation to the frequency domain:

$$
F(\omega)=2 \frac{E^{*}(\omega)}{\left(1-v^{2}\right)} \sqrt{R \delta_{0}} \delta(\omega)
$$

where $F(\omega)$ and $\delta(\omega)$ are the force and indentation signals in the frequency domain, respectively, $E^{*}(\omega)$ is the complex Young's modulus, $v$ is the Poisson's ratio assumed to be 
$0.5, R$ is the radius of the indenter and $\omega$ is the angular frequency. The complex shear modulus $G^{*}(\omega)$ can be written using $G^{*}(\omega)=\frac{E^{*}(\omega)}{2(1+v)} 98$ :

$$
G^{*}(\omega)=G^{\prime}(\omega)+i G^{\prime \prime}(\omega)=\frac{(1-v)}{4 \sqrt{R \delta_{0}}} \frac{F(\omega)}{\delta(\omega)}
$$

where $G^{\prime}(\omega)$ is the storage modulus and $G^{\prime \prime}(\omega)$ is the loss modulus. The ratio of the force $F(\omega)$ and indentation $\delta(\omega)$ is calculated from the measured amplitudes $A^{F}(\omega)$ and $A^{\delta}(\omega)$ and the phase shifts $\theta^{F}(\omega)$ and $\theta^{\delta}(\omega)$ of the oscillatory signals ${ }^{99}$ :

$$
\frac{F(\omega)}{\delta(\omega)}=\frac{A^{F}(\omega)}{A^{\delta}(\omega)} e^{i\left(\theta^{F}(\omega)-\theta^{\delta}(\omega)\right)}
$$

where the difference of the phase shifts $\left(\theta^{F}(\omega)-\theta^{\delta}(\omega)\right)$ is in the range of $0^{\circ}$ (elastic solid) and $90^{\circ}$ (viscous fluid). Furthermore, the hydrodynamic drag contribution on the cantilever oscillation was estimated and subtracted from the complex shear modulus as previously described ${ }^{100}$ :

$$
G^{*}(\omega)=\frac{(1-v)}{4 \sqrt{R \delta_{0}}}\left[\frac{F(\omega)}{\delta(\omega)}-i \omega b(0)\right],
$$

where $b(h)$ is the hydrodynamic drag coefficient function measured from non-contact oscillations of the cantilever at different distances $h$ from the sample, and $b(0)$ is the extrapolation to distance 0 from the sample. For PNP-TR-TL cantilevers, the hydrodynamic drag coefficient was estimated to be $b(0)=5.28 \mu \mathrm{N} \mathrm{s} \mathrm{m}^{-1}$.

\section{PERTURBATION EXPERIMENTS}

\section{CAV1 knock-down}

For RNAi experiments, cells were transfected using RNAiMax reagent (13778030, Thermo Fisher Scientific) and a reverse transfection protocol. Per transfection, $200 \mathrm{ng}$ of esiRNA (Eupheria Biotech, Germany) or 300 ng of ON-TARGETplus siRNA (Dharmacon, CO, USA) and $2 \mu \mathrm{l}$ RNAiMax were prepared in OptiMEM (31985062, Gibco) according to the manufacturer's instructions and pipetted onto 12-well plates (see Supplementary Table 6 for full list of siRNAs used). Cells in $1 \mathrm{ml}$ of culture medium were plated on top of the transfection mix at 
a density allowing for sub-confluent growth within the experimental timeframe. $72 \mathrm{~h}$ post transfection, cells were collected for the mechanical characterization and Western blot analysis.

\section{Plasmid for CAV1 overexpression}

The cDNA of CAV1 was amplified by PCR, introducing Nhel and Xhol restriction sites in the flanking regions. The PCR product was then cloned into the PCGIT destination vector (a kind gift from P. Serup, University of Copenhagen, Denmark) under the CAG promoter and with dTomato fluorescent marker under internal ribosomal entry site (IRES) downstream of CAV1.

\section{Transient CAV1 overexpression in ECC4 and TGBC cells}

ECC4 and TGBC cells were transiently transfected with the CAV1 overexpression plasmid by electroporation (Neon Transfection System, MPK5000, Thermo Fisher Scientific). Per transfection $0.3 \times 10^{6}$ ECC4 cells, or $0.2 \times 10^{6}$ TGBC cells were mixed with $1 \mu \mathrm{g}$ of plasmid DNA in PBS. Electroporation was conducted using $10 \mu$ I Neon tips (MPK1096, Thermo Fisher Scientific) and a program of two pulses of $1050 \mathrm{~V}$ and $30 \mathrm{~ms}$ duration each. Electroporated cells were transferred to $500 \mu \mathrm{l}$ of fresh culture medium in a 24-well plate. The cells were collected for mechanical characterization and Western blot analysis $72 \mathrm{~h}$ post transfection. To identify fluorescent cells during mechanical characterization, the combined real-time fluorescence and deformability cytometry (RT-FDC) ${ }^{73}$ setup was used, and the maximum intensity of the fluorescence signal from channel 2 (excitation 561 nm, 10\% laser power; collection 700/75) was utilized for gating.

\section{Transient CAV1 overexpression in MCF10A-ER-src cells}

MCF10A-ER-src cells were transiently transfected with the CAV1 overexpressing plasmid using Effectene transfection reagent (301425, Qiagen). One day before transfection, cells were seeded on glass bottom 35-mm dishes (FluoroDish; FD35100, WPI, FL, USA) at a density of 20,000 cells per well. Transfection was performed according to the manufacturer's instruction using $75 \mu \mathrm{l}$ EC buffer, $0.6 \mu \mathrm{g}$ plasmid DNA, $4.8 \mu$ l Enhancer and $6 \mu$ l Effectene 
reagent per well. $24 \mathrm{~h}$ post transfection cells were induced with $1 \mu \mathrm{M}$ TAM. Mechanical analysis was performed after additional $72 \mathrm{~h}$ of culture.

\section{Western blotting}

For Western blot analysis of carcinoma and MCF10A-ER-Src cell lines, cell pellets were collected in parallel with mechanical measurements and lysed using ice-cold RIPA buffer (89900, ThermoFisher Scientific) supplemented with protease/phosphatase inhibitor cocktail (78441, ThermoFisher Scientific) and benzonase (E1014, Sigma-Aldrich). The lysates were cleared at $4^{\circ} \mathrm{C}$ by 10 -minute sonication followed by 10 -minute centrifugation at $16,900 \mathrm{~g}$. Obtained supernatants were mixed with Laemmli buffer (final concertation: 62.5 mM Tris$\mathrm{HCl}$ (pH 6.8), 2\% SDS, 10\% glycerol, 5\% $\beta$-mercaptoethanol, 0.01\% bromophenol blue), boiled $\left(5 \mathrm{~min}\right.$ at $\left.95^{\circ} \mathrm{C}\right)$, and separated by SDS-PAGE electrophoresis on 4-20\% gradient gels (MiniPROTEAN TGX Precast Gels; 4561093, Biorad, CA, USA) in MOPS SDS Running buffer (B0001, ThermoFisher Scientific). After transferring the proteins onto a PVDF membrane (Merck Millipore), the membranes were blocked in TBS-T (20 mM Tris, $137 \mathrm{mM} \mathrm{NaCl}, 0.1 \%$ Tween) containing 5\% w/v skimmed milk powder (T145.1, Carl Roth, Germany) for 40 minutes. Next, membranes were incubated with the primary anti-Cav1 (1:1000; D46G3; \#3267, Cell Signaling Technology, MA, USA) and anti-GAPDH (1:5000; ab9485, Abcam, UK) antibodies at $4^{\circ} \mathrm{C}$ overnight in 5\% milk/TBS-T, washed, and incubated with anti-rabbit HRP-conjugated secondary antibody (1:4000; ab97069, Abcam). Chemiluminescence detection was performed using Pierce Enhanced Chemi-Luminescence (ECL) substrate (32109, ThermoFisher Scientific) and ECL films (GE28-9068-37, Merck Millipore). Films were developed in an OptiMax X-ray film processor (KODAK, NY, USA). Quantitative analysis was performed on scanned films using the gel analysis tool in Jmage/ version 2.0.0-rc-69/1.52p (https://imagej.nih.gov/). For western blot analysis of MEFs the same anti-Cav1 antibody (1:1000; D46G3; \#3267, Cell Signaling) was used, and anti-tubulin antibody (1:2000; DM1A; \#3873, Cell Signaling) was used as a loading control. Goat anti-mouse 680 and goat antirabbit 800 (1:2000; A28183 and A32735, ThermoFisher Scientific) antibodies were used for 
secondary detection. Membranes were scanned with the Odyssey imaging system (LI-COR Biosciences, NE, USA).

\section{COMPUTATIONAL ANALYSIS}

\section{Transcriptomic datasets}

Transcriptomic datasets were retrieved from online databases (Gene Expression Omnibus, GEO and DNA Data Bank of Japan, DDBJ) with accession numbers listed in Table 1. Overview of experimental detail for RNA profiling procedures and data analysis in individual datasets is presented in Supplementary Table 7. The IDs of samples used in respective categories in each dataset are listed in Supplementary Table 8. In case of multiple entries for the same gene in a given transcriptomic dataset, the expression values were averaged, so that only one entry per gene and sample was available.

\section{PC-corr analysis}

Before performing the PC-corr analysis, the glioblastoma and iPSC datasets were intersected and normalized by taking the log10 (glioblastomna) or zscore (iPSC) of the subset of 9,452 overlapping genes. The PC-corr analysis was conducted on individual datasets as described in detail elsewhere ${ }^{41}$. In brief, PCA was performed using svd function in MATLAB (R2020a, MathWorks, MA, USA) on normalized datasets. The original PC loadings from the component providing good separation of sample categories (PC1 for both analyzed datasets) were processed in a two-step procedure including the normalization and scaling. The processing of the PC loadings is performed to adjust the distribution of the loadings to the range of Pearson's correlation values $[-1,1]$, so that they are comparable when computing the $P C$-corr value. The normalization was performed using a custom function developed previously ${ }^{41}$ of the following formula:

$$
V_{i}^{*}=\operatorname{sgn}\left(V_{i}^{0}\right) \log _{10}\left(1+\frac{\left|V_{i}^{0}\right|}{\left\langle\left|V^{0}\right|\right\rangle}\right)
$$


where $V_{i}^{*}$ denotes the normalized loading corresponding to the $i$-th feauture, $V_{i}^{0}$ the original loading corresponding to the $i$-th feauture, and $\left\langle\left|V^{0}\right|\right\rangle$ the average of all absolute loadings of the vector $V^{0}$.

The normalized loadings were then scaled to fall on the interval $[-1,1]$ using a previously developed custom function ${ }^{41}$ :

$$
V_{i}=\operatorname{sgn}\left(V_{i}^{*}\right) \frac{\left|V_{i}^{*}\right|-\min \left(\left|V^{*}\right|\right)}{\max \left(\left|V^{*}\right|\right)-\min \left(\left|V^{*}\right|\right)}
$$

where $V_{i}$ denotes the processed loading corresponding to the $i$-th feature, and $\left|V^{*}\right|$ the vector containing absolute values of all normalized loadings.

The $P C$-corr values for each pair of features were computed according to Equation 1. The PC-corr results of the glioblastoma and iPSC datasets were combined as described in the results section. Gene pairs showing different $P C$-corr signs were masked by setting the $P C$-corr ${ }^{c o m b}$ to zero. The genes and edges comprising the network were obtained via thresholding strategies described in the main text. The network was visualized using cytoscape (cytoscape 3.8.0; https://cytoscape.org/) ${ }^{101}$.

\section{Statistical analysis}

The RT-DC datasets were compared using generalized linear mixed effects models with the aid of ShapeOut (ShapeOut 1.0.1; https://github.com/ZELLMECHANIK-DRESDEN/ShapeOut; Zellmechanik Dresden) as described in detail elsewhere ${ }^{102}$. AFM datasets were compared using two-sided Wilcoxon rank sum test in MATLAB (R2020a, MathWorks). Western blot results were compared using a two-sided two-sample t-test in MATLAB (R2020a, MathWorks). 


\section{Acknowledgements}

We thank Isabel Richter and Christine Schweitzer for technical assistance, Miguel Sanchez (CNIC, Spain) and Konstantinos Anastasiadis (TU Dresden, Germany) for helpful discussions, Len R. Stephens (Babraham Institute, UK) for provision of MCF10A PIK3CA cells, and Kevin Struhl (Harvard Medical School, MA, USA) for provision of MCF10A ER-Src cells. We further thank the Microstructure Facility at the Center for Molecular and Cellular Bioengineering $(\mathrm{CMCB})$ at the Technische Universität Dresden (in part funded by the State of Saxony and the European Regional Development Fund) for hosting the chip fabrication. The authors acknowledge funding from the Alexander von Humboldt-Stiftung (Alexander von Humboldt Professorship to J.G.), European Commission (ERC Starting Grant "LightTouch" \#282060 to J.G.), Marie Sklodowska-Curie Actions under the European Union's Horizon 2020 research and innovation programme (BIOPOL ITN, \#641639 to M.A.d.P. and J.G.), Deutsche Forschungsgemeinschaft (\#GU 612/5-1 and \#399422891 to J.G.), Comunidad Autónoma de Madrid (Tec4Bio-CM, \#S2018/NMT-4443 to M.A.d.P.), and Fundació La Marató de TV3 (\#201936-30-31 to M.A.d.P). A.T. is a fellow of the Mildred Scheel Early Career Center Dresden (MSNZ) funded by the German Cancer Aid (Deutsche Krebshilfe).

\section{Author contributions}

J.G. and C.V.C. conceived the project. Y.G. under supervision of C.V.C. performed the computational analysis on transcriptomics data. M.U., M.W., M.H., M.K., and N.T. performed the measurements of mechanical states. M.U. and M.W. performed the genetic manipulation experiments. S.A. and A.T. provided methodological support with AFM measurements and data analysis. J.D. and O.F. were involved in MCF10A PIK3CA experiments. M.D. under supervision of F.C. isolated the developing neurons. F.L. under supervision of M.A.d.P. performed MEF experiments. M.U. and M.W. analyzed the experimental data. M.U. visualized the data and prepared figures. M.U., with the support of J.G., C.V.C., and Y.G. prepared the initial version of the manuscript. All authors revised and edited the manuscript. J.G., C.V.C., M.A.d.P. and A.T. acquired funding. 


\section{Competing interests}

The authors declare no competing interest.

\section{Data availability statement}

The transcriptomic data used in this study were obtained from public repositories under accession numbers listed in Table 1. The mechanical characterization data are available on figshare (https://doi.org/10.6084/m9.figshare.c.5399826).

\section{Code availability}

The MATLAB code for performing the PC-corr analysis was based on the code deposited alongside a previous publication ${ }^{41}$, accessible on GitHub https://github.com/biomedicalcybernetics/PC-corr net. 


\section{References}

1. Di Carlo, D. A Mechanical Biomarker of Cell State in Medicine. J. Lab. Autom. 17, 32-42 (2012).

2. Guck, J. \& Chilvers, E. R. Mechanics meets medicine. Sci. Transl. Med. 5, 3-6 (2013).

3. Nematbakhsh, Y. \& Lim, C. T. Cell biomechanics and its applications in human disease diagnosis. Acta Mech. Sin. 31, 268-273 (2015).

4. Guck, J. Some thoughts on the future of cell mechanics. Biophysical Reviews vol. 11 667670 (2019).

5. Lecuit, T. \& Lenne, P. F. Cell surface mechanics and the control of cell shape, tissue patterns and morphogenesis. Nat. Rev. Mol. Cell Biol. 8, 633-644 (2007).

6. Mammoto, T. \& Ingber, D. E. Mechanical control of tissue and organ development. Development 137, 1407-20 (2010).

7. Stooke-Vaughan, G. A. \& Campàs, O. Physical control of tissue morphogenesis across scales. Curr. Opin. Genet. Dev. 51, 111-119 (2018).

8. Hannezo, E. \& Heisenberg, C. P. Mechanochemical Feedback Loops in Development and Disease. Cell 178, 12-25 (2019).

9. Huang, S. \& Ingber, D. E. Cell tension, matrix mechanics, and cancer development. Cancer Cell 8, 175-176 (2005).

10. Suresh, S. Biomechanics and biophysics of cancer cells. Acta Mater. 55, 3989-4014 (2007).

11. Kumar, S. \& Weaver, V. M. Mechanics, malignancy, and metastasis: The force journey of a tumor cell. Cancer Metastasis Rev. 28, 113-127 (2009).

12. Wirtz, D., Konstantopoulos, K. \& Searson, P. C. The physics of cancer: The role of physical interactions and mechanical forces in metastasis. Nat. Rev. Cancer 11, 512-522 (2011). 
13. Gensbittel, V. et al. Mechanical Adaptability of Tumor Cells in Metastasis. Dev. Cell 56, 164-179 (2021).

14. Fletcher, D. A. \& Mullins, R. D. Cell mechanics and the cytoskeleton. Nature 463, 485$492(2010)$.

15. Salbreux, G., Charras, G. \& Paluch, E. Actin cortex mechanics and cellular morphogenesis. Trends Cell Biol. 22, 536-545 (2012).

16. Chugh, P. \& Paluch, E. K. The actin cortex at a glance. J. Cell Sci. 131, 1-9 (2018).

17. Kelkar, M., Bohec, P. \& Charras, G. Mechanics of the cellular actin cortex: From signalling to shape change. Curr. Opin. Cell Biol. 66, 69-78 (2020).

18. Wang, N. \& Stamenović, D. Contribution of intermediate filaments to cell stiffness, stiffening, and growth. Am. J. Physiol. - Cell Physiol. 279, 188-194 (2000).

19. Seltmann, K., Fritsch, A. W., Käs, J. A. \& Magin, T. M. Keratins significantly contribute to cell stiffness and impact invasive behavior. Proc. Natl. Acad. Sci. U. S. A. 110, 1850718512 (2013).

20. Mendez, M. G., Restle, D. \& Janmey, P. A. Vimentin enhances cell elastic behavior and protects against compressive stress. Biophys. J. 107, 314-323 (2014).

21. Patteson, A. E. et al. Vimentin protects cells against nuclear rupture and DNA damage during migration. J. Cell Biol. 218, 4079-4092 (2019).

22. Stankevicins, L. D. C. et al. Vimentin provides the mechanical resilience required for amoeboid migration and protection of the nucleus. bioRxiv 720946 (2019) doi:10.1101/720946.

23. Kubitschke, $\mathrm{H}$. et al. Actin and microtubule networks contribute differently to cell response for small and large strains. New J. Phys. 19, (2017).

24. Chang, Y. C., Nalbant, P., Birkenfeld, J., Chang, Z. F. \& Bokoch, G. M. GEF-H1 couples nocodazole-induced microtubule disassembly to cell contractility via RhoA. Mol. Biol. Cell 19, 2147-2153 (2008). 
25. Zhou, E. H. et al. Universal behavior of the osmotically compressed cell and its analogy to the colloidal glass transition. Proc. Natl. Acad. Sci. 106, 10632-10637 (2009).

26. Moeendarbary, E. et al. The cytoplasm of living cells behaves as a poroelastic material. Nat. Mater. 12, 253-261 (2013).

27. Guo, M. et al. Cell volume change through water efflux impacts cell stiffness and stem cell fate. Proc. Natl. Acad. Sci. c, 201705179 (2017).

28. Caille, N., Thoumine, O., Tardy, Y. \& Meister, J. J. Contribution of the nucleus to the mechanical properties of endothelial cells. J. Biomech. 35, 177-187 (2002).

29. Serrano-Alcalde, F., García-Aznar, J. M. \& Gómez-Benito, M. J. The role of nuclear mechanics in cell deformation under creeping flows. J. Theor. Biol. 432, 25-32 (2017).

30. Chugh, P. et al. Actin cortex architecture regulates cell surface tension. Nat. Cell Biol. 19, 689-697 (2017).

31. Toyoda, Y. et al. Genome-scale single-cell mechanical phenotyping reveals diseaserelated genes involved in mitotic rounding. Nat. Commun. 8, 1-10 (2017).

32. Rosendahl, P. et al. Real-time fluorescence and deformability cytometry. Nat. Methods 15, 355 (2018).

33. Wang, J., Lü, D., Mao, D. \& Long, M. Mechanomics: An emerging field between biology and biomechanics. Protein Cell 5, 518-531 (2014).

34. Putra, V. D. L. et al. Mechanomics Approaches to Understand Cell Behavior in Context of Tissue Neogenesis, During Prenatal Development and Postnatal Healing. Front. Cell Dev. Biol. 7, 1-8 (2020).

35. Zhang, F. et al. Mechanomics analysis of hESCs under combined mechanical shear, stretch, and compression. Biomech. Model. Mechanobiol. (2020) doi:10.1007/s10237020-01378-5.

36. Lang, M. Lighting up the mechanome. Front. Eng. Reports leading-edge Eng. from 2007 Symp. (2008). 
37. Van Loon, J. J. W. A. Mechanomics and physicomics in gravisensing. Microgravity Sci. Technol. 21, 159-167 (2009).

38. Song, M. J. et al. Mapping the mechanome of live stem cells using a novel method to measure local strain fields in situ at the fluid-cell interface. PLoS One 7, 1-12 (2012).

39. Song, M. J., Dean, D. \& Knothe Tate, M. L. Mechanical modulation of nascent stem cell lineage commitment in tissue engineering scaffolds. Biomaterials 34, 5766-5775 (2013).

40. Wang, H. et al. Mechanomics Biomarker for Cancer Cells Unidenti fi able through Morphology and Elastic Modulus. (2021) doi:10.1021/acs.nanolett.1c00003.

41. Ciucci, S. et al. Enlightening discriminative network functional modules behind principal component analysis separation in differential-omic science studies. Sci. Rep. 7, 1-24 (2017).

42. Poser, S. W. et al. Controlling distinct signaling states in cultured cancer cells provides a new platform for drug discovery. FASEB J. 33, 9235-9249 (2019).

43. Urbanska, M. et al. Single-cell mechanical phenotype is an intrinsic marker of reprogramming and differentiation along the mouse neural lineage. Development 144, 4313-4321 (2017).

44. Otto, O. et al. Real-time deformability cytometry: on-the-fly cell mechanical phenotyping. Nat. Methods 12, 199-202 (2015).

45. Hanley, J. A. \& McNeil, B. J. The meaning and use of the area under a receiver operating characteristic (ROC) curve. Radiology 143, 29-36 (1982).

46. Mokbel, M. et al. Numerical Simulation of Real-Time Deformability Cytometry To Extract Cell Mechanical Properties. ACS Biomater. Sci. Eng. (2017) doi:10.1021/acsbiomaterials.6b00558.

47. Brenner, B., Tang, L. H., Klimstra, D. S. \& Kelsen, D. P. Small-cell carcinomas of the gastrointestinal tract: A review. J. Clin. Oncol. 22, 2730-2739 (2004). 
48. Kalemkerian, G. P. et al. Small cell lung cancer: Clinical practice guidelines in oncology. JNCCN J. Natl. Compr. Cancer Netw. 11, 78-98 (2013).

49. Arulmozhivarman, G. et al. Zebrafish In-Vivo Screening for Compounds Amplifying Hematopoietic Stem and Progenitor Cells: - Preclinical Validation in Human CD34+ Stem and Progenitor Cells. Sci. Rep. 7, 1-15 (2017).

50. Juvin, V. et al. Signaling via Class IA Phosphoinositide 3-Kinases (PI3K) in Human, Breast-Derived Cell Lines. PLoS One 8, (2013).

51. Bader, A. G., Kang, S. \& Vogt, P. K. Cancer-specific mutations in PIK3CA are oncogenic in vivo. Proc. Natl. Acad. Sci. U. S. A. 103, 1475-1479 (2006).

52. Kang, S., Bader, A. G. \& Vogt, P. K. Phosphatidylinositol 3-kinase mutations identified in human cancer are oncogenic. Proc. Natl. Acad. Sci. U. S. A. 102, 802-807 (2005).

53. Tonge, P. D. et al. Divergent reprogramming routes lead to alternative stem-cell states. Nature 516, 192-197 (2014).

54. Aprea, J. et al. Transcriptome sequencing during mouse brain development identifies long non-coding RNAs functionally involved in neurogenic commitment. EMBO J. 32, 3145-3160 (2013).

55. Forrest, A. R. R. et al. A promoter-level mammalian expression atlas. Nature 507, 462470 (2014).

56. Kiselev, V. Y. et al. Perturbations of PIP3 signalling trigger a global remodelling of mRNA landscape and reveal a transcriptional feedback loop. Nucleic Acids Res. 43, 9663-9679 (2015).

57. Zhang, B. \& Horvath, S. Statistical Applications in Genetics and Molecular Biology A General Framework for Weighted Gene Co- Expression Network Analysis A General Framework for Weighted Gene Co- Expression Network Analysis. Stat. Appl. Genet. Mol. Biol. 4, Article17 (2005).

58. J., R., A.K., D. \& W., Z. A general co-expression network-based approach to gene 
expression analysis: Comparison and applications. BMC Syst. Biol. 4, (2010).

59. Parton, R. G. \& Del Pozo, M. A. Caveolae as plasma membrane sensors, protectors and organizers. Nat. Rev. Mol. Cell Biol. 14, 98-112 (2013).

60. Lolo, F.-N. et al. Continuous membrane tension buffering by caveolin-1 invaginations switch to discontinuous caveolae flattening by PTRF. Prep.

61. Sinha, B. et al. Cells respond to mechanical stress by rapid disassembly of caveolae. Cell 144, 402-413 (2011).

62. Raudenska, M., Gumulec, J., Balvan, J. \& Masarik, M. Caveolin-1 in oncogenic metabolic symbiosis. Int. J. Cancer 147, 1793-1807 (2020).

63. Pol, A., Morales-Paytuví, F., Bosch, M. \& Parton, R. G. Non-caveolar caveolins - Duties outside the caves. J. Cell Sci. 133, (2020).

64. Johannessen, M., Møler, S., Hansen, T., Moens, U. \& Van Ghelue, M. The multifunctional roles of the four-and-a-half-LIM only protein FHL2. Cell. Mol. Life Sci. 63, 268-284 (2006).

65. Nakazawa, N., Sathe, A. R., Shivashankar, G. V. \& Sheetz, M. P. Matrix mechanics controls FHL2 movement to the nucleus to activate p21 expression. Proc. Natl. Acad. Sci. U. S. A. 113, E6813-E6822 (2016).

66. Sun, X. et al. Mechanosensing through Direct Binding of Tensed F-Actin by LIM Domains. Dev. Cell (2020) doi:10.1016/j.devcel.2020.09.022.

67. Winkelman, J. D., Anderson, C. A., Suarez, C., Kovar, D. R. \& Gardel, M. L. Evolutionarily diverse LIM domain-containing proteins bind stressed actin filaments through a conserved mechanism. Proc. Natl. Acad. Sci. U. S. A. 117, 25532-25542 (2020).

68. Jin, L., Shen, F., Weinfeld, M. \& Sergi, C. Insulin Growth Factor Binding Protein 7 (IGFBP7)-Related Cancer and IGFBP3 and IGFBP7 Crosstalk. Front. Oncol. 10, 1-14 (2020).

69. Liu, R., Hossain, M. M., Chen, X. \& Jin, J. P. Mechanoregulation of SM22a/Transgelin. Biochemistry 56, 5526-5538 (2017). 
70. Dvorakova, M., Nenutil, R. \& Bouchal, P. Transgelins, cytoskeletal proteins implicated in different aspects of cancer development. Expert Rev. Proteomics 11, 149-165 (2014).

71. Adams, J. C. \& Lawler, J. The thrombospondins. Cold Spring Harb. Perspect. Biol. 3, 1-29 (2011).

72. Huang, T., Sun, L., Yuan, X. \& Qiu, H. Thrombospondin-1 is a multifaceted player in tumor progression. Oncotarget 8, 84546-84558 (2017).

73. Rosendahl, P. et al. Real-time fluorescence and deformability cytometry - flow cytometry goes mechanics. Nat. Methods.

74. Hirsch, H. A., Iliopoulos, D., Tsichlis, P. N. \& Struhl, K. Metformin selectively targets cancer stem cells, and acts together with chemotherapy to block tumor growth and prolong remission. Cancer Res. 69, 7507-7511 (2009).

75. Tavares, S. et al. Actin stress fiber organization promotes cell stiffening and proliferation of pre-invasive breast cancer cells. Nat. Commun. 8, (2017).

76. Hirsch, H. A. et al. A Transcriptional Signature and Common Gene Networks Link Cancer with Lipid Metabolism and Diverse Human Diseases. Cancer Cell 17, 348-361 (2010).

77. Urbanska, M. et al. A comparison of microfluidic methods for high-throughput cell deformability measurements. Nat. Methods 17, 587-593 (2020).

78. Durán, C. et al. Nonlinear machine learning pattern recognition and bacteriametabolite multilayer network analysis of perturbed gastric microbiome. Nat. Commun. 12, 1926 (2021).

79. Lin, H.-H. et al. Mechanical phenotype of cancer cells: cell softening and loss of stiffness sensing. Oncotarget 6, (2015).

80. Moreno-Vicente, R. et al. Caveolin-1 Modulates Mechanotransduction Responses to Substrate Stiffness through Actin-Dependent Control of YAP. Cell Rep. 25, 16221635.e6 (2018). 
81. Strippoli, R. et al. Caveolin1 and YAP drive mechanically induced mesothelial to mesenchymal transition and fibrosis. Cell Death Dis. 11, 1-19 (2020).

82. Dupont, S. et al. Role of YAP/TAZ in mechanotransduction. Nature 474, 179-184 (2011).

83. Rausch, V. et al. The Hippo Pathway Regulates Caveolae Expression and Mediates Flow Response via Caveolae. Curr. Biol. 29, 242-255.e6 (2019).

84. Stein, C. et al. YAP1 Exerts Its Transcriptional Control via TEAD-Mediated Activation of Enhancers. PLoS Genet. 11, 1-28 (2015).

85. Zhao, B. et al. TEAD mediates YAP-dependent gene induction and growth control. Genes Dev. 22, 1962-1971 (2008).

86. Jiang, W. R. et al. Mechanoregulation of h2-calponin gene expression and the role of notch signaling. J. Biol. Chem. 289, 1617-1628 (2014).

87. Hossain, M. M., Smith, P. G., Wu, K. \& Jin, J. P. Cytoskeletal tension regulates both expression and degradation of h2-calponin in lung alveolar cells. Biochemistry 45, 15670-15683 (2006).

88. Hossain, M. M., Crish, J. F., Eckert, R. L., Lin, J. J. C. \& Jin, J. P. H2-Calponin Is Regulated By Mechanical Tension and Modifies the Function of Actin Cytoskeleton. J. Biol. Chem. 280, 42442-42453 (2005).

89. Goetz, J. G., Lajoie, P., Wiseman, S. M. \& Nabi, I. R. Caveolin-1 in tumor progression: The good, the bad and the ugly. Cancer Metastasis Rev. 27, 715-735 (2008).

90. Wang, Z. et al. Caveolin-1, a stress-related oncotarget, in drug resistance. Oncotarget 6, 37135-37150 (2015).

91. Nawaz, A. A. et al. Intelligent image-based deformation-assisted cell sorting with molecular specificity. Nat. Methods 17, 595-599 (2020).

92. Lv, J. et al. Cell softness regulates tumorigenicity and stemness of cancer cells. 1-14 (2020) doi:10.15252/embj.2020106123. 
93. Arulmozhivarman, G. et al. In Vivo Chemical Screen in Zebrafish Embryos Identifies Regulators of Hematopoiesis Using a Semiautomated Imaging Assay. J. Biomol. Screen. 1087057116644163 (2016) doi:10.1177/1087057116644163.

94. Razani, B. et al. Caveolin-1 Null Mice Are Viable but Show Evidence of Hyperproliferative and Vascular Abnormalities. J. Biol. Chem. 276, 38121-38138 (2001).

95. Urbanska, M., Rosendahl, P., Kräter, M. \& Guck, J. High-throughput single-cell mechanical phenotyping with real-time deformability cytometry. Methods Cell Biol. 147, (2018).

96. Sneddon, I. N. The relation between load and penetration in the axisymmetric boussinesq problem for a punch of arbitrary profile. Int. J. Eng. Sci. 3, 47-57 (1965).

97. Alcaraz, J. et al. Microrheology of human lung epithelial cells measured by atomic force microscopy. Biophys. J. (2003) doi:10.1016/S0006-3495(03)75014-0.

98. Landau, L. D., Lifshitz, E. M., Sykes, J. B., Reid, W. H. \& Dill, E. H. Theory of Elasticity: Vol. 7 of Course of Theoretical Physics. Phys. Today 13, 44-46 (2009).

99. Rother, J., Nöding, H., Mey, I. \& Janshoff, A. Atomic force microscopy-based microrheology reveals significant differences in the viscoelastic response between malign and benign cell lines. Open Biol. (2014) doi:10.1098/rsob.140046.

100. Alcaraz, J. et al. Correction of microrheological measurements of soft samples with atomic force microscopy for the hydrodynamic drag on the cantilever. Langmuir (2002) doi:10.1021/la0110850.

101. Shannon, P. et al. Cytoscape: A Software Environment for Integrated Models of Biomolecular Interaction Networks. 2498-2504 doi:10.1101/gr.1239303.metabolite.

102. Herbig, M., Mietke, A., Müller, P. \& Otto, O. Statistics for real-time deformability cytometry: Clustering, dimensionality reduction, and significance testing. Biomicrofluidics 12, (2018). 